\title{
Psychiatric comorbidities in adult patients with epilepsy (A systematic review)
}

\author{
RALUCA SIMONA GURGU ${ }^{1 *}$, ADELA MAGDALENA CIOBANU ${ }^{2,3^{*}}$, \\ ROXANA IONELA DANASEL ${ }^{3}$ and CRISTINA AURA PANEA ${ }^{1,4^{*}}$

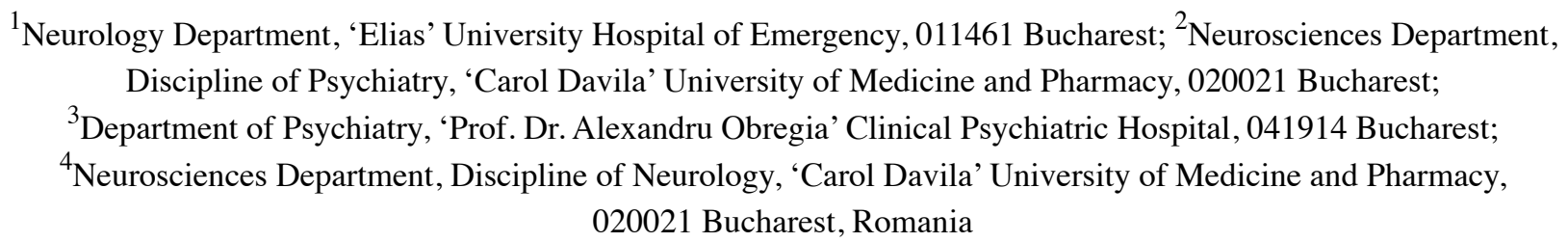

Received April 27, 2021; Accepted May 27, 2021

DOI: $10.3892 /$ etm.2021.10341

\begin{abstract}
The co-occurrence of epilepsy and psychiatric disorders is long known. The scope of this systematic review was to describe the prevalence of specific interictal psychiatric disorders in patients with epilepsy and to assess possible associations between psychiatric disorders and other sociodemographic or clinical characteristics of epilepsy patients. MEDLINE and ScienceDirect were searched for original articles published between January 2015 and February 2021 describing studies that involved epilepsy patients with psychiatric comorbidities. We identified 13 studies with heterogenous methodology and reporting. Prevalence of any psychiatric disorder observed was up to $51 \%$ in idiopathic generalized epilepsy (IGE), up to $43.1 \%$ in temporal lobe epilepsy (TLE) and up to $43.3 \%$ in a general population of patients with epilepsy. The most frequent psychiatric comorbidities associated with epilepsy included $\mathrm{mood} / \mathrm{affective} \mathrm{disorders} \mathrm{(up} \mathrm{to} 40 \%$ for lifetime occurrence and up to $23 \%$ for current occurrence), anxiety disorders (up to $30.8 \%$ for lifetime occurrence and up to $15.6 \%$ for current occurrence), personality disorders [up to $11 \%$ in juvenile myoclonic epilepsy (JME)] and psychotic disorders (up to $4 \%$ of epilepsy patients). In focal epilepsy, depressive disorders might be associated with specific brain imaging findings and with cognitive impairment. Anxiety disorders are associated with a higher frequency of generalized tonico-clonic
\end{abstract}

Correspondence to: Dr Adela Magdalena Ciobanu, Neurosciences Department, Discipline of Psychiatry, 'Carol Davila' University of Medicine and Pharmacy, 37 Dionisie Lupu Street, 020021 Bucharest, Romania

E-mail: adela.ciobanu@gmail.com

*Contributed equally

Key words: psychiatric disorders, mood disorders, anxiety disorders, psychotic disorders, personality disorders, epilepsy, seizures seizure (GTCS) and with worse social functioning. Psychotic disorders were found to be associated with longer duration of epilepsy. Childhood maltreatment experiences were found to be a powerful predictor for the occurrence of psychiatric comorbidities in epilepsy patients, while data regarding association of other epilepsy characteristics with the presence of psychiatric disorders is conflicting.

\section{Contents}

1. Introduction

2. Methods

3. Results

4. Discussion and limitations

5. Conclusions

\section{Introduction}

The co-occurrence of psychiatric disorders with epilepsy has been long known and debated, as the relationship between the two pathologies is complex and bidirectional $(1,2)$. The recent International League Against Epilepsy (ILAE) guidelines recommend early and continued screening, diagnosis and management for all epilepsy-associated comorbidities in every patient with epilepsy (3). However, the prevalence of specific psychiatric comorbidities and correlation with other clinical characteristics of epilepsy is still a matter of debate, reflecting different methodologies in research studies and clinical practice $(4,5)$ and the changing diagnostic criteria for both epilepsy (6) and psychiatric disorders (7).

As far as we are aware, to date there are only a few systematic reviews concerning psychiatric disorders associated with epilepsy. We were not able to find recent reviews that address the prevalence of most common psychiatric disorders in patients with epilepsy. A systematic review published in 2016 addressed the risk factors for depression in community treated epilepsy cases (based on research results published between 2000 and 2013) and found that sociodemographic variables 
(such as patient age and sex) played the most important role (8). Another older systematic review focused on psychosocial predictors of depression and anxiety in epilepsy patients (9), while more recent reviews assessed mainly screening and/or diagnostic methods for depression (10) or anxiety (11).

The aim of this study was to describe the prevalence of specific interictal psychiatric disorders (depressive disorders, anxiety disorders, psychotic disorders and personality disorders) in patients with epilepsy and to compare this to the prevalence of psychiatric disorders in the general population and to assess possible associations between psychiatric disorders and other sociodemographic or clinical characteristics of epilepsy patients.

\section{Methods}

This review was performed according to the recommendations of the Preferred Reporting Items of Systematic Reviews and Meta-Analyses (PRISMA) statement (12).

Search strategy. A systematic literature search using two electronic databases, namely MEDLINE and ScienceDirect was conducted in February 2021. The databases were searched using a combination of keywords: Depression, anxiety, affective disorder, psychotic disorder, psychosis, personality, traits, epilepsy and seizures. The search was limited to original research conducted in humans, published in English language between January 2015 and February 2021. We applied additional filters for age (adults, older than 18 years).

Study inclusion criteria. Only controlled studies (randomized, cohort, cross-sectional or case-control) were included in this analysis. Studies were selected if conducted in patients diagnosed with epilepsy/unprovoked single seizure, if the diagnostic methods used for epilepsy and psychiatric disorders were reported, if the characterization of both epilepsy and psychiatric comorbidities was considered adequate, and if methodology (including statistical analysis) was clearly described.

Study exclusion criteria. We excluded studies assessing only ictal/peri-ictal psychiatric manifestations, treatment response or adverse effects. We also excluded studies assessing psychogenic non-epileptic seizures that did not report data of psychogenic non-epileptic seizure patient groups separately, studies using for diagnosis questionnaires administered online/remotely, studies that evaluated psychiatric disorders only with self-administered screening tools and studies that did not include comparative groups. Articles that did not meet the primary inclusion criteria were also excluded (e.g.: Case reports, case series, reviews, abstracts, book chapters, patents, and conference papers) due to the lack of data for assessment and comparison.

Data extraction. The data extracted from the eligible studies included: Study setting (including year of publication, type of research facility and country where the study was conducted), study type, study objectives, inclusion and exclusion criteria, total number of subjects included, mean/median age of patients included, information on each comparative group (description, number of patients, mean/median age of patients), information on epilepsy (diagnosis criteria used, epilepsy syndrome, duration of epilepsy, current seizure frequency), information on antiepileptic and psychiatric drugs used by patients at the time of inclusion in the study, information on psychiatric disorders observed (screening tools used, confirmation tests applied, raters' qualification, diagnosis code as per Statistical Manual of Mental Disorders IV/Diagnostic and Statistical Manual for Psychiatric Disorders-5th edition (DSM-5, 2013), statistical methods, results (prevalence of psychiatric disorders, statistically significant associations with other clinical or paraclinical features of epilepsy) and limitations of each study.

Study quality assessment. The risk of bias for the articles selected for this review was assessed with the following quality checking instruments: The RoB 2.0 tool for randomized trials (13), Newcastle-Ottawa Scale (NOS) for Cohort and Case-Control Studies, respectively (14), Joanna Briggs Institute (JBI) Critical Appraisal Checklist for analytical cross-sectional studies (15) and Crombie's Items for purely descriptive cross-sectional studies.

\section{Results}

Results of the search. Using the search criteria described above we identified 3,986 original articles (1,550 articles in MEDLINE database and 2,436 articles in ScienceDirect database). We assessed 258 full articles for eligibility. Finally, we included in this analysis 13 articles. The flowchart shown in Fig. 1 details this process.

Description of the studies. This analysis includes 13 research studies, conducted in 7 countries (Australia, one study; Brazil, three studies; China, two studies; Germany, two studies; Japan, one study; Spain, one study; and USA, three studies). We included data obtained from one randomized controlled trial (assessing depression and anxiety improvement in epilepsy patients when multidisciplinary management was applied) because the severity of the psychiatric disorders in the control group was assessed after 12 months (16), two cohort studies [one designed to detect depression disorders and anxiety disorders in adult patients with childhood onset epilepsy (17) and the other one analyzing psychotic disorders in idiopathic focal epilepsy (18)], one case-control study that evaluated specifically the psychiatric outcome in drug-resistant epilepsy patients who underwent surgery vs. patients that were considered non-eligible for the procedure (because baseline data on psychiatric disorders diagnosed was solid and because the patients in the control group were re-assessed by psychiatrist at 6 months, thus providing information about the time evolution of psychiatric disorders in people with epilepsy) (19), four studies with a cross sectional design [first assessing interictal personality in patients with juvenile myoclonic epilepsy (JME) and in patients with mesial temporal lobe epilepsy related to hippocampal sclerosis (MTLE/HS)] (20), second one analyzing interictal dysphoric disorder and interictal personality in drug-resistant temporal lobe epilepsy (TLE) (21), the third one evaluating depressive symptoms and executive functions in patients with TLE (22) and the last one on white matter changes in patients with TLE and depression (23). We selected 


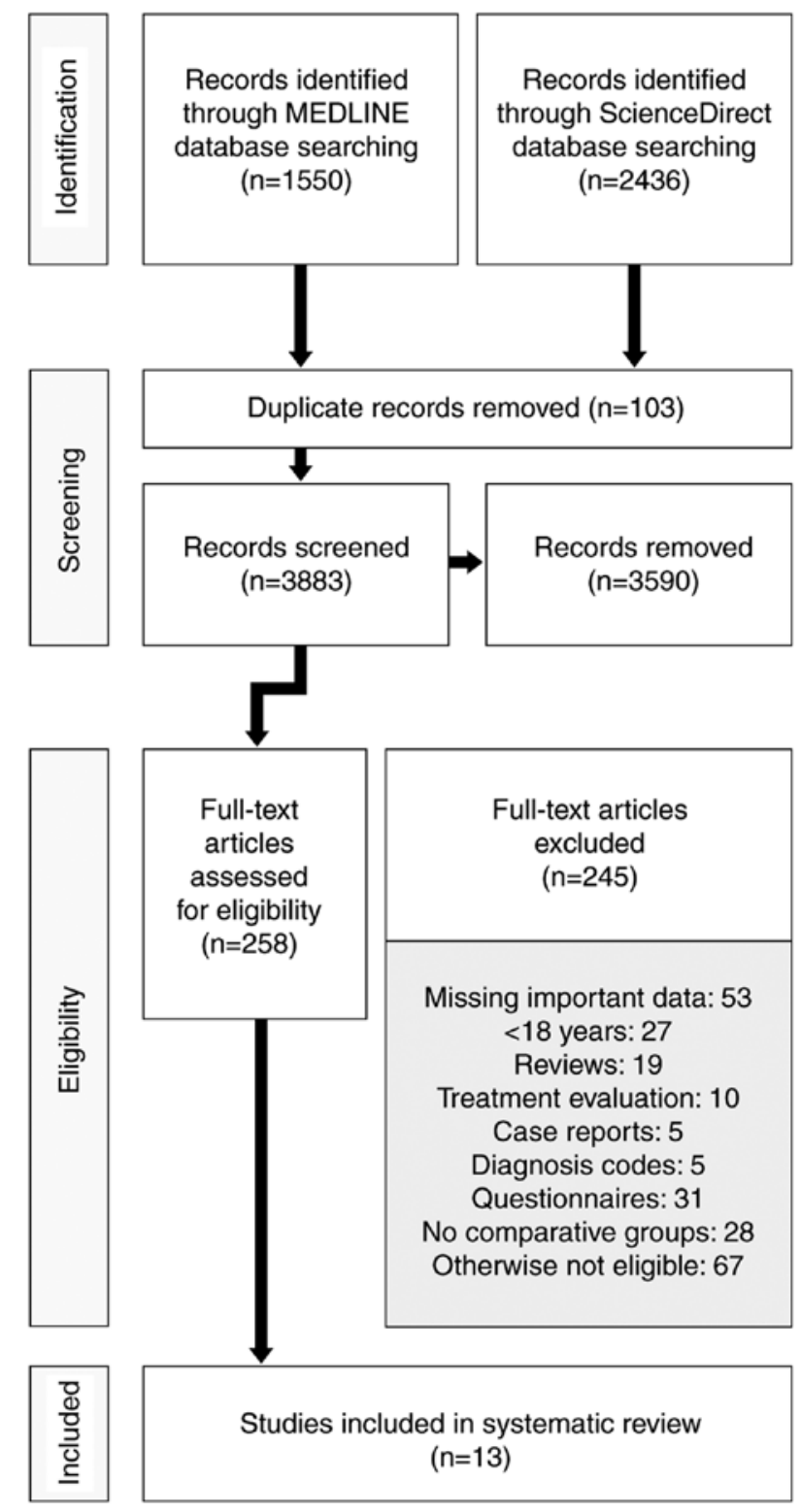

Figure 1. PRISMA Flow Diagram of the study selection process.

for this analysis five studies that assessed the relation between interictal dysphoric disorder and other psychiatric disorders in patients with epilepsy (24), the association between childhood trauma and psychiatric comorbidities in epilepsy (25), social adjustment determinants in JME (26), possible phenotypes of depression in epilepsy (27) and brainstem sonographic changes in patients with idiopathic generalized epilepsy (IGE) (28). Importantly, we identified two studies having the same first author $(24,25)$ and two studies performed by the same research team $(22,23)$ that had different research questions, but used a shared sample population of epilepsy patients that was compared with a different control group in the first case and with the same control group (but fewer subjects) in the second case.

In total, 2,192 epilepsy patients were analyzed (1,252 as sample cases and 940 in some of the control groups). Most studies (nine) included patients investigated or followed up in dedicated epilepsy units (of which at least six were tertiary referral centres, as per authors' descriptions), while only four studies included patients from general hospitals/practice. A summary of the selected studies is shown in Table I.

The best represented epilepsy type was TLE (eight studies included patients with this type of epilepsy), followed by focal extratemporal lobe epilepsy (ETLE) and IGE (five studies each). Two articles mentioned the inclusion of patients with generalized seizures (without any reference to the actual epilepsy diagnosis in these patients) and another three studies included patients with other (not mentioned) types of epilepsy or presenting unspecified or unclassifiable epilepsy/seizures.

Duration of epilepsy (mean/median) was less than 10 years in three studies $(16,18,28)$, between 10 and 20 years in six studies $(18,20,24-27)$ and longer than 20 years in three studies (20-22). Three research articles did not contain clear information on epilepsy duration.

Seizure frequency and antiepileptic drugs used for treatment are difficult to summarize, because different reporting measures were used. A summary of the epilepsy characteristics in the selected studies is shown in Table II.

Psychiatric disorders. The self-assessment tools used for the screening or quantification of psychiatric symptoms in the studies we analyzed were: Neurobehavior Inventory (NBI), Revised Personality Inventory (NEO-PI-R) (both tools assess personality traits), Interictal Dysphoric Disorder Inventory (IDDI) (evaluates interictal dysphoric disorder symptoms over the last 12 months), Neurological Depression Disorder Inventory for Epilepsy (NDDI-E) (created to detect current depression symptoms in epilepsy patients), Beck Depression Inventory (BDI) and Beck Depression Inventory-II (BDI-II) (for symptoms of depression), Hospital Anxiety and Depression Scale (HADS), State-Trait Anxiety Inventory (STAI), Patient Health Questionnaire-Generalized Anxiety Disorder-7 Items (PHQ-GAD-7) and Beck Depression Inventory (BAI) (the last three tools rate current anxiety symptoms).

Psychiatric disorders were confirmed according to the DSM IV criteria in eight studies, using different structured or semi-structured clinical interviews such as Structured Clinical Interview for DSM IV Axis I disorders (SCID I) (two studies) $(20,27)$, Structured Clinical Interview for DSM IV Axis II disorders (SCID II) (one study) (20), SCID for DSM-IV Clinician Version (SCID CV) (one study) (19), MINI International Neuropsychiatric Interview (M.I.N.I.) (two studies) (22,23), Mini International Neuropsychiatric Interview Plus (M.I.N.I.-Plus) (two studies) $(24,25)$, Structured Clinical Interview for DSM-IV (SCID) (without further details, one study) (28). DSM IV-TR criteria were used for diagnosis in two studies: In one study Diagnostic Interview Survey (DIS-IV) was applied by phone in subjects and in one control group, while the Composite International Diagnostic Interview (CIDI) was used for the second control group (17); in the second study SCID for Axis I and II was applied (26). Finally, DSM-5 criteria for diagnosis were utilized in one study only (the method used for diagnosis was standard psychiatric assessment, without a formal standardized interview) (21). Diagnosis criteria employed by the psychiatrist who confirmed the psychiatric diagnoses were not specified at all in two studies $(16,18)$. The professional confirming the psychiatric diagnosis was a psychiatrist in two studies $(19,20)$ and psychologist in two studies $(22,23)$. The remaining seven 


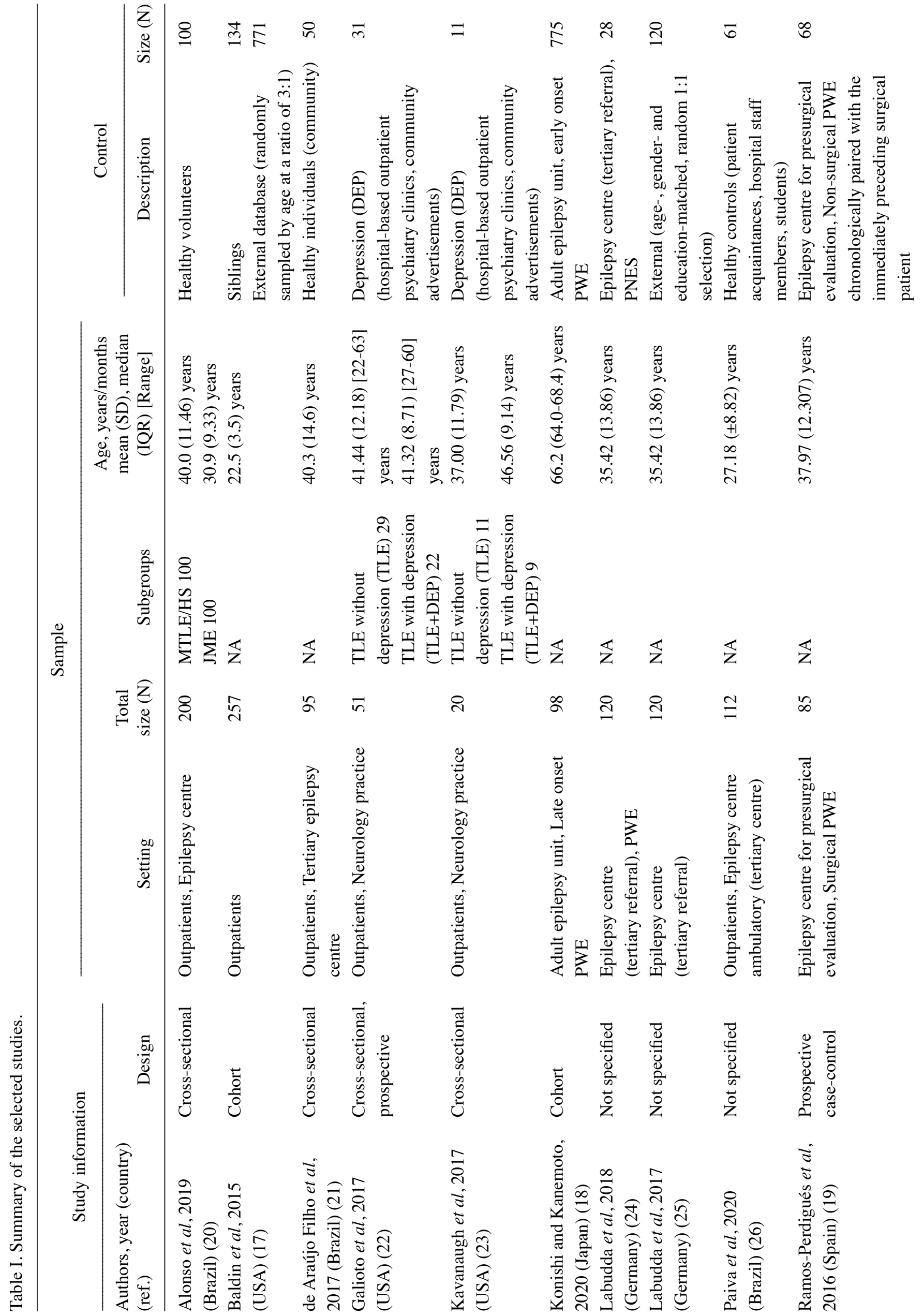


articles did not clearly specify the qualifications of the person(s) who conducted the psychiatric interview $(17,21,24-28)$.

The prevalence of any psychiatric disorder was high for all epilepsy types: 43 and 51\%, respectively, in two populations with JME $(20,26), 33 \%$ in patients with MTLE/HS that underwent surgery (20), $43.1 \%$ in patients with pharmacoresistant TLE-MTS (21) and $43.3 \%$ in a sample of patients with epilepsy from a tertiary referral centre $(24,25)$. A summary of the psychiatric disorders characteristics is shown in Table III.

Mood/affective disorders. Prevalence of current mood disorders (major depressive episode, dysthymic disorder, manic episode, hypomanic episode during the previous year) was $12.8 \%$ in adults with neurotypical childhood onset epilepsy (comparison with both control groups-siblings and external controls-was not statistically significant) (17). Current major depressive episode was present in $18.2 \%$ of the patients from a population with TLE and depression (22). In a general population of epilepsy patients from a third referral centre, $21.7 \%$ were diagnosed with at least one affective disorder $(24,25)(5 \%$ presented major depressive episode, $9.2 \%$ recurrent depressive disorder and $7.5 \%$ dysthymia, while none of these patients was diagnosed with bipolar disorder), without statistical significance when prevalence was compared with psychogenic non-epileptic seizures patients in the control group (24). In focal epilepsy (mostly pharmacoresistant) current major depressive episode or other depressive disorders (major depressive disorder, minor depressive disorder, dysthymia) had a prevalence of $23 \%$ (27).

Lifetime mood disorders (major depressive episode, major depressive disorder single episode, major depressive disorder recurrent, depressive episode with melancholic features, bipolar I disorder, bipolar II disorder, bipolar I disorder single manic episode, dysthymic disorder, manic episode, mixed episode, hypomanic episode) had a prevalence of $20.6 \%$ in adults with childhood-onset epilepsy, statistically not significant when compared with the prevalence of lifetime mood disorders in both control groups (sibling and external controls) (17). The prevalence of lifetime history of depression in focal epilepsy (mostly pharmacoresistant) was 40\% (27).

Prevalence of depressive disorder (not specified if lifetime/current) was 16 and 9\%, respectively, in two populations of patients with juvenile myoclonic epilepsy $(20,26)$ and $26 \%$ in patients with MTLE/HS, late post-surgically (20). Prevalence of major depressive disorder was $22.1 \%$ in patients with pharmacoresistant TLE-MTS, significantly higher than in healthy individuals from the community (21). Prevalence of affective disorders (including major depressive episodes, recurrent depression, dysthymic disorder, affective disorder due to a medical condition or substance disorder, adjustment disorder and bipolar disorder, but not specified if lifetime/current) was $22 \%$ in pharmacoresistant epilepsy patients assessed for epilepsy surgery (19). Finally, the incidence of depression (no further details regarding the exact DSM IV diagnosis or if current/lifetime) was $34.78 \%$ in patients with idiopathic generalized epilepsy (with tonic-clonic seizures) (IGE TCS) (28).

Suicidal risk and suicidal attempt, evaluated by Diagnostic Interview Survey for Children (DISC-IV), in adults with childhood-onset epilepsy had a prevalence of 16 and 5.1\%, 


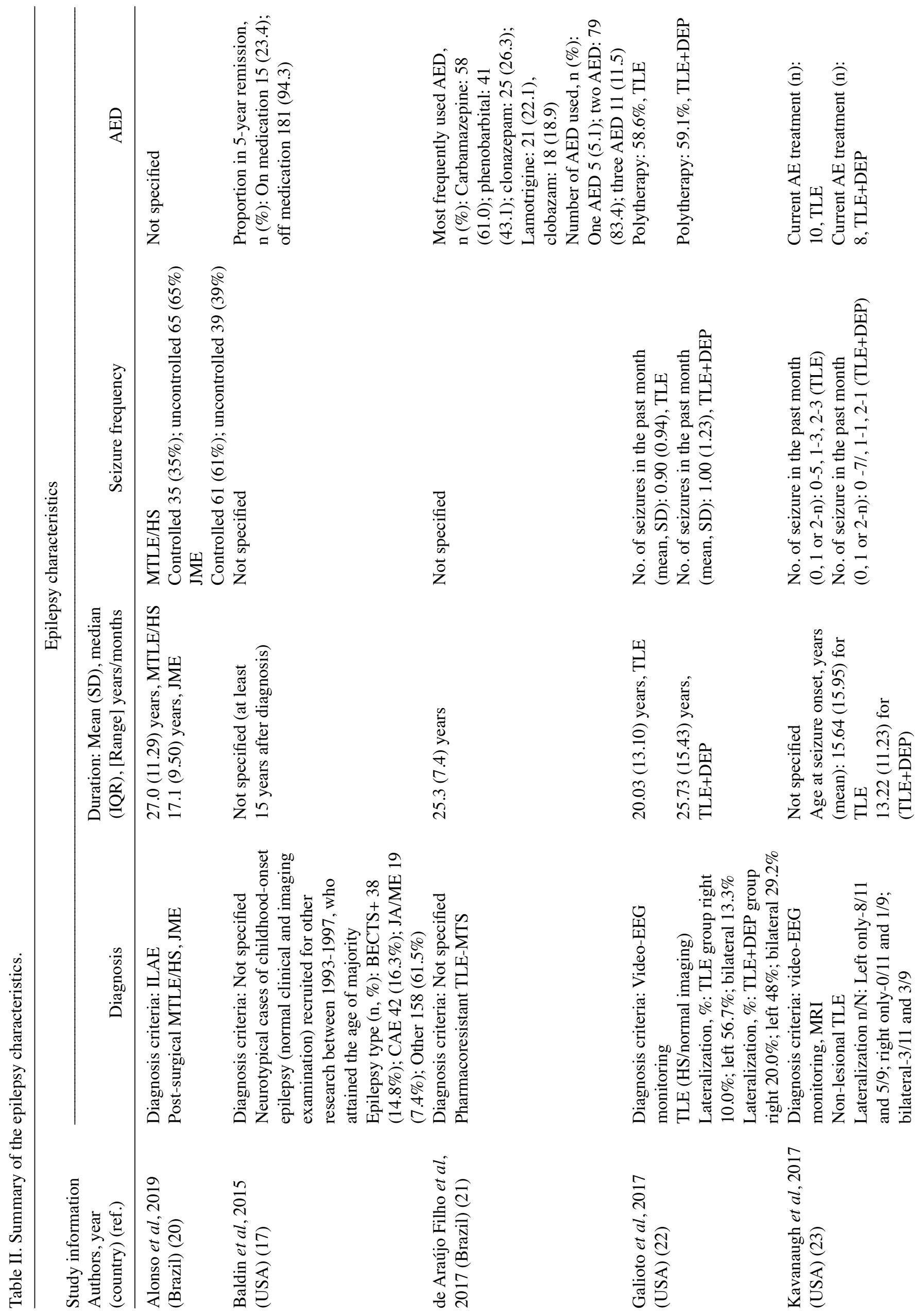




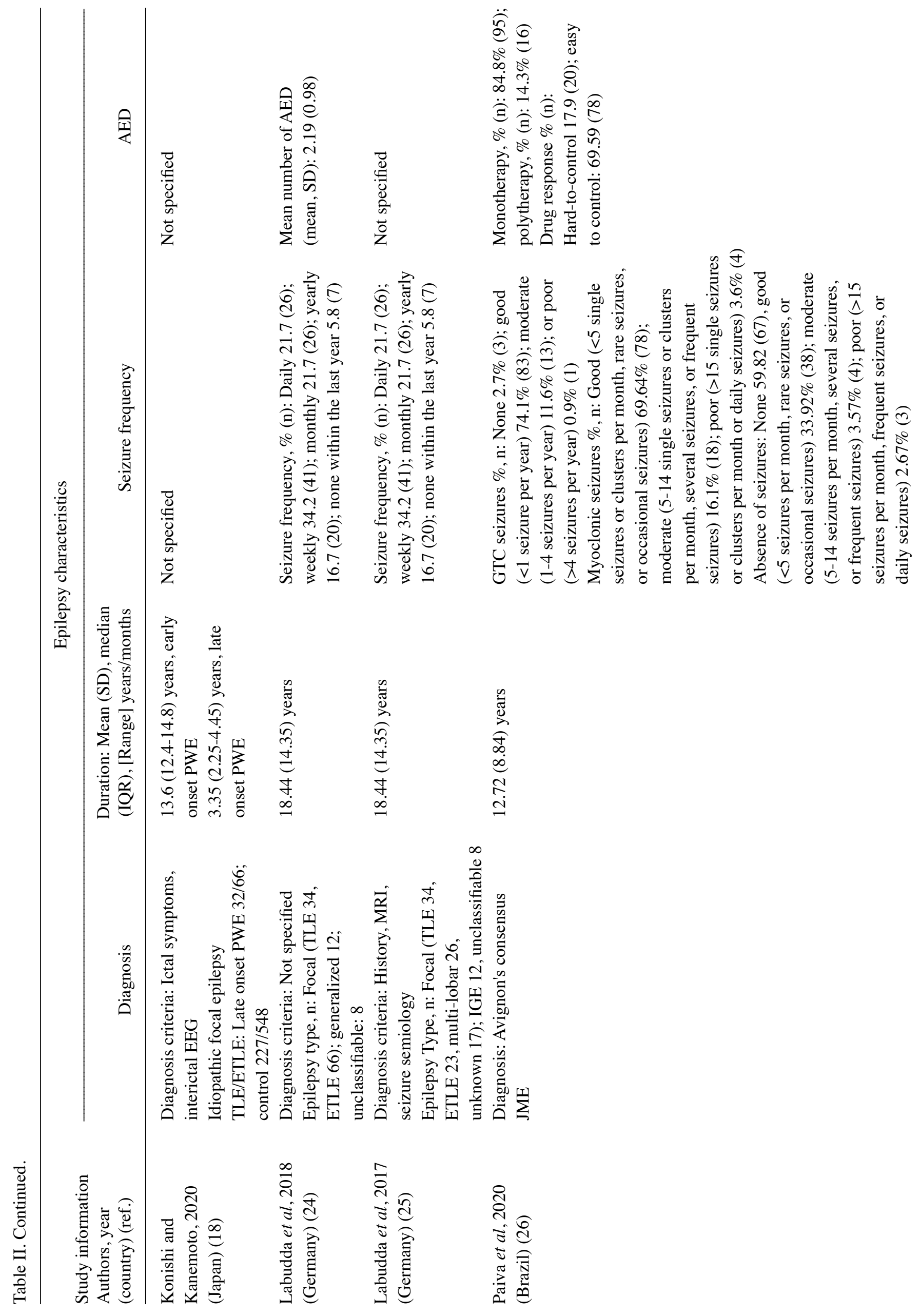




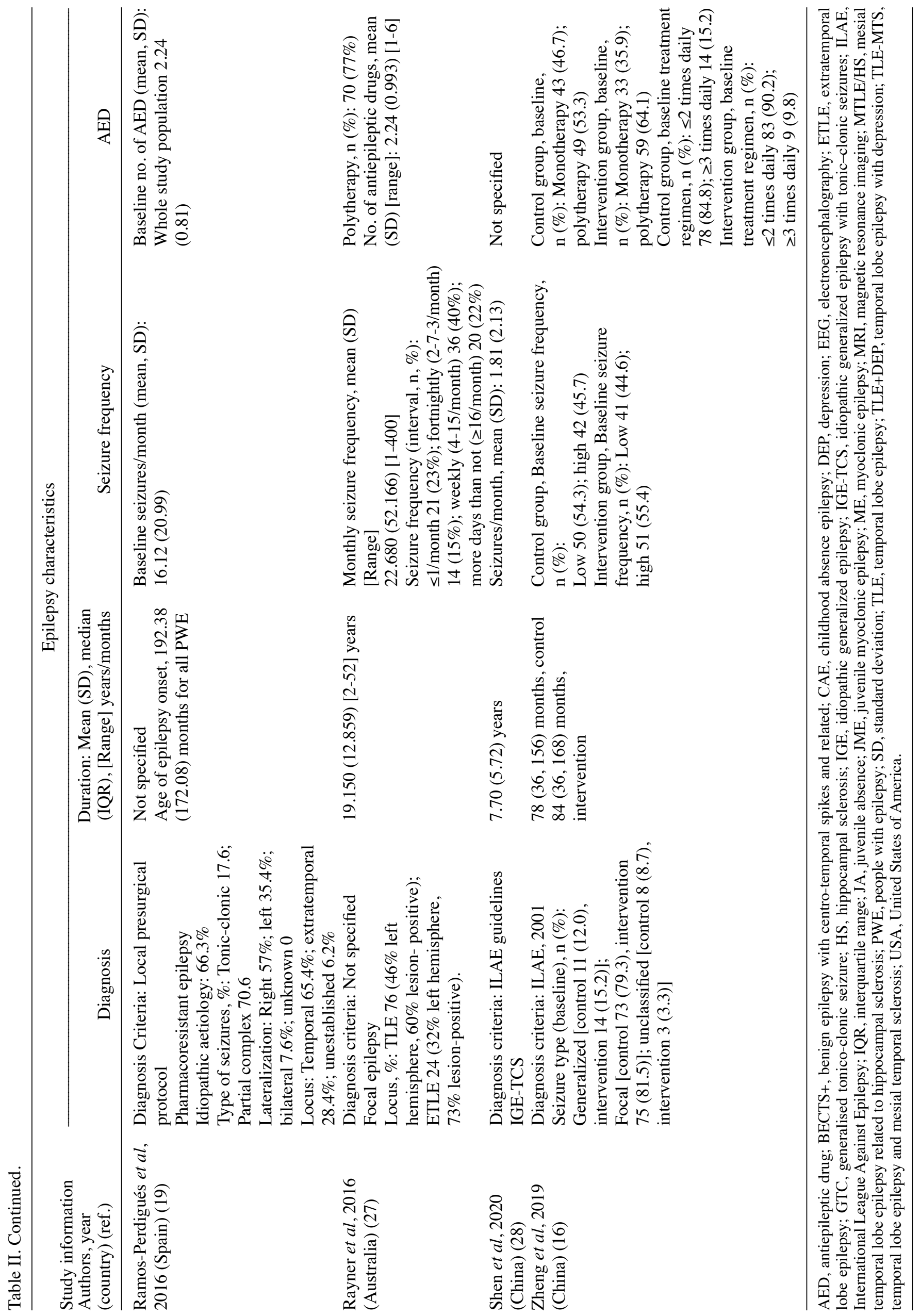




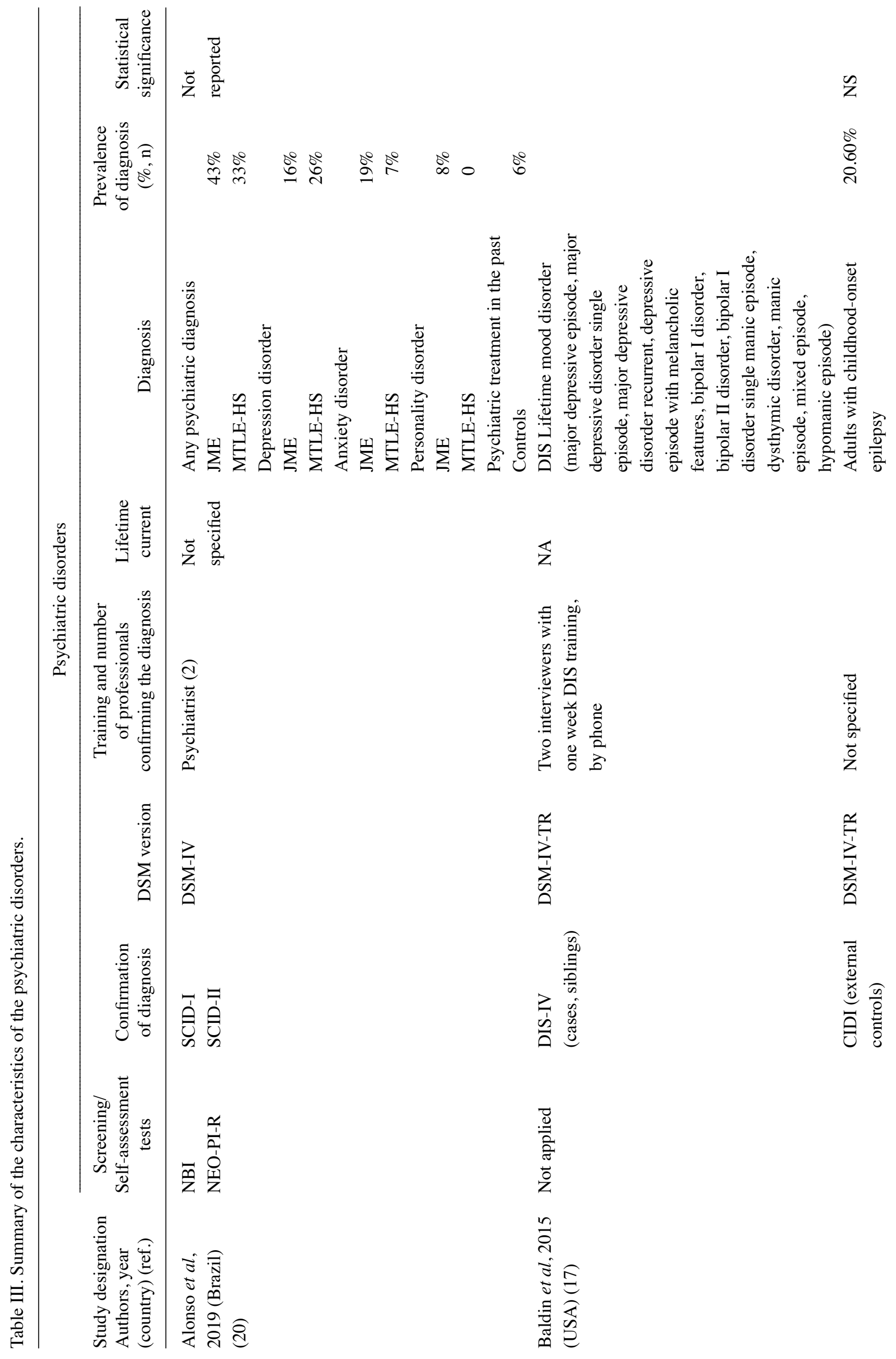




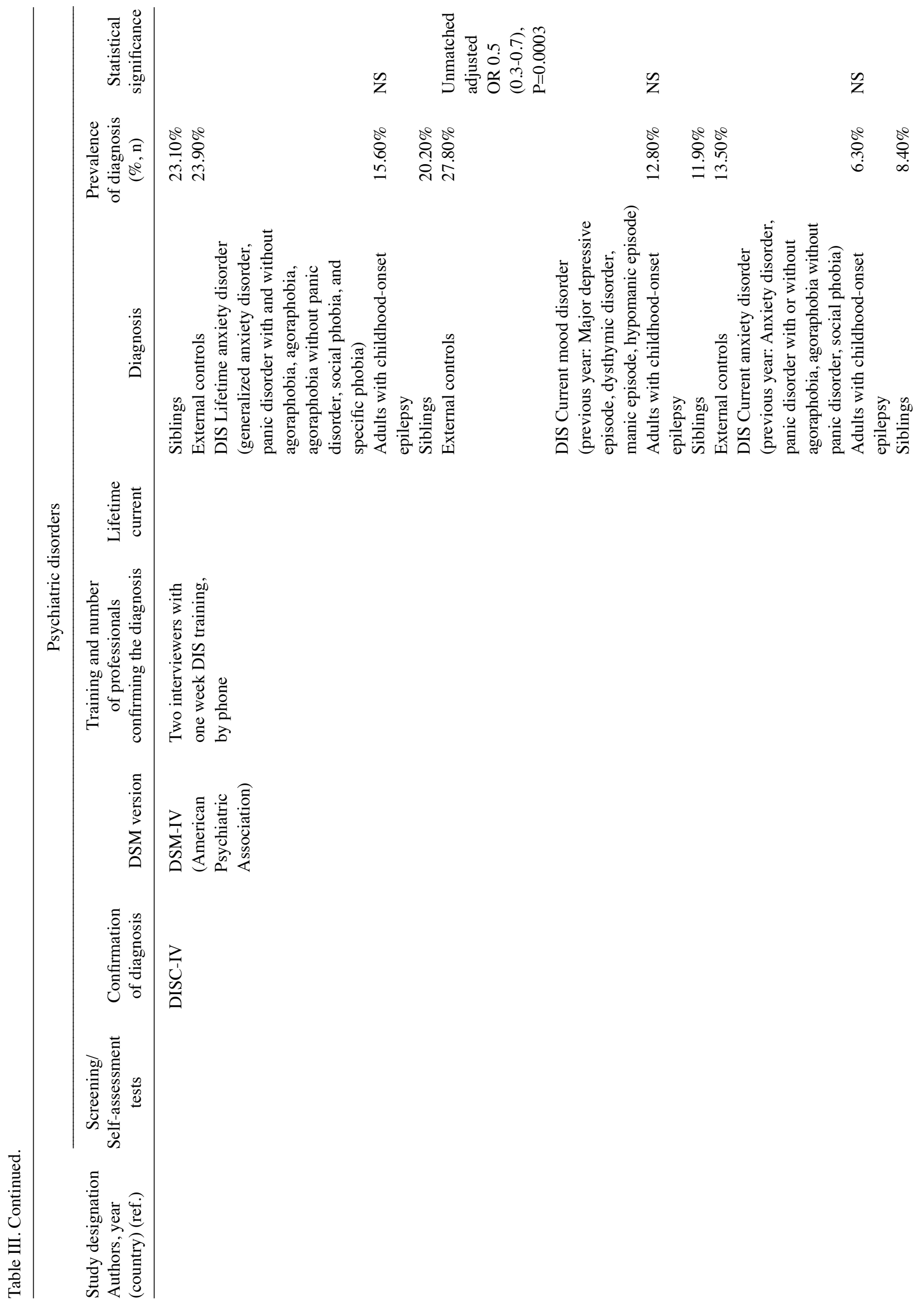




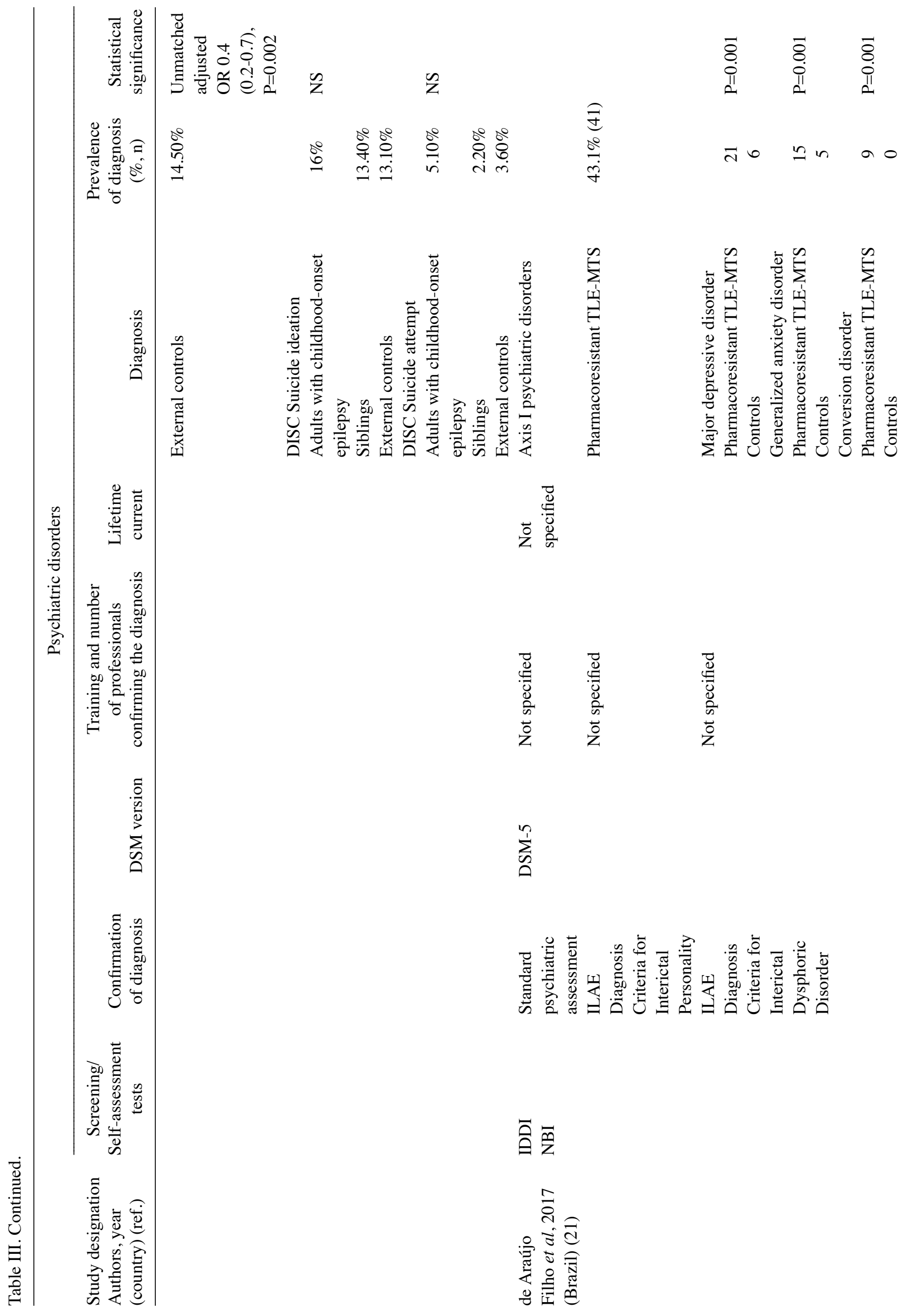




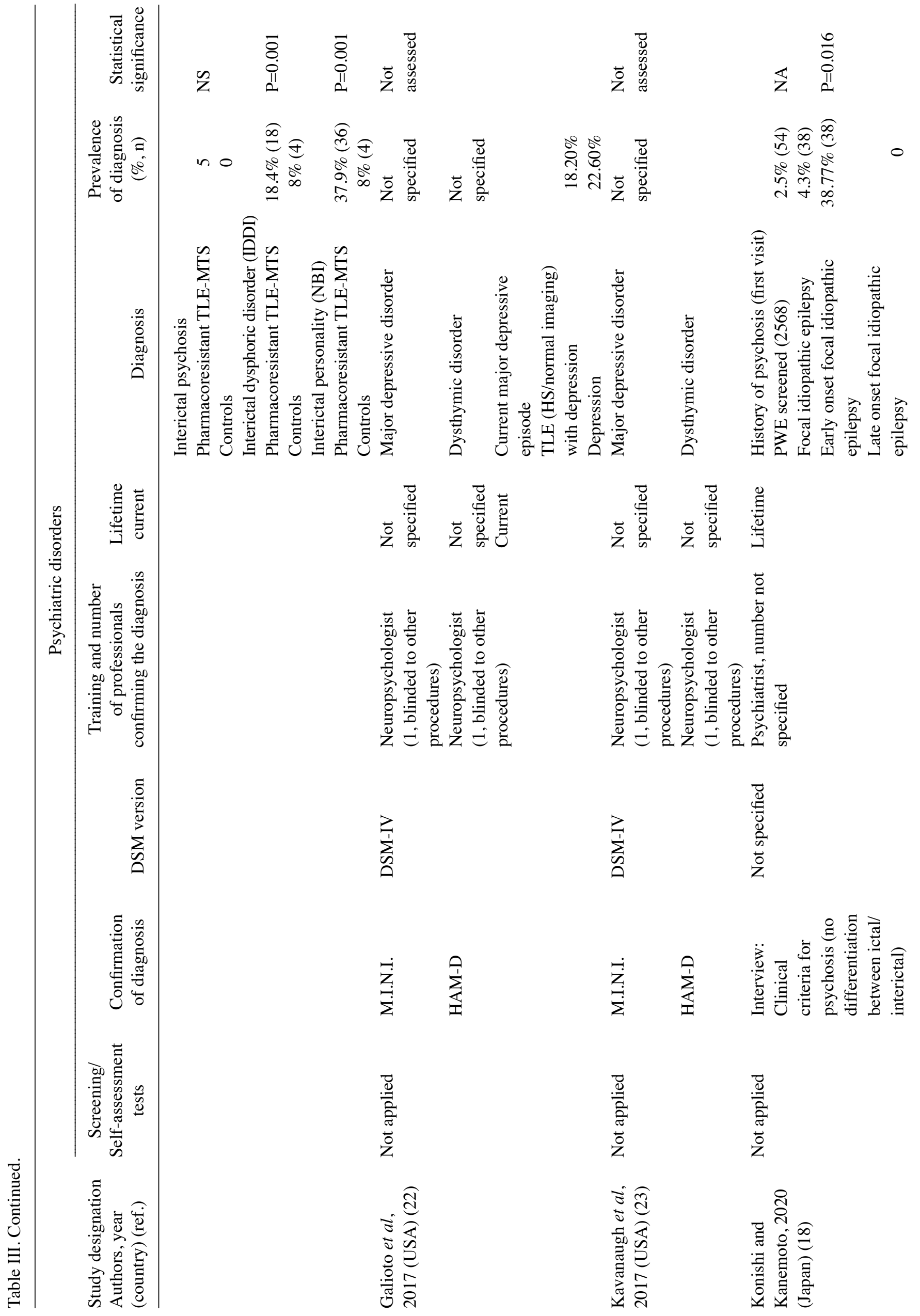




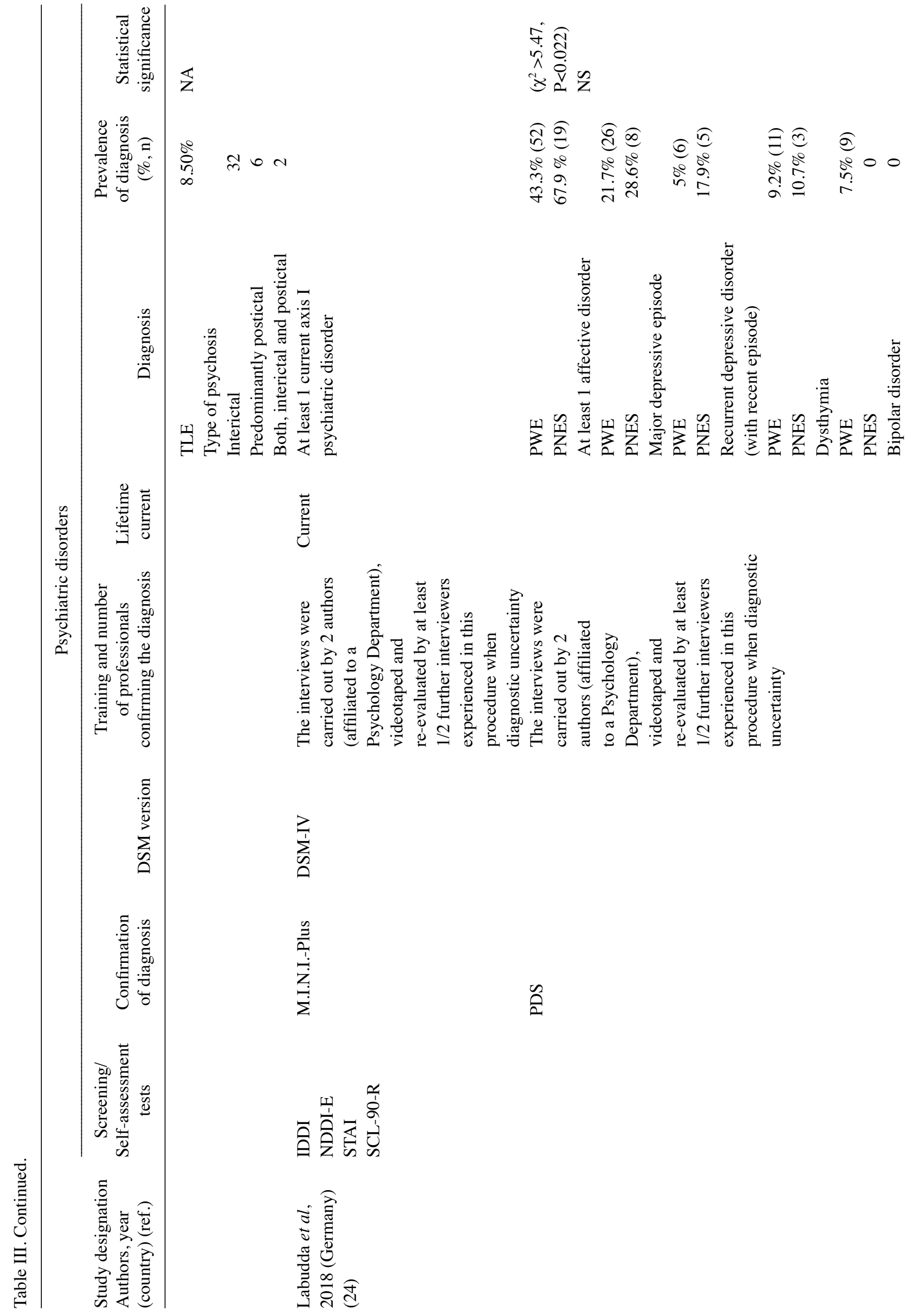




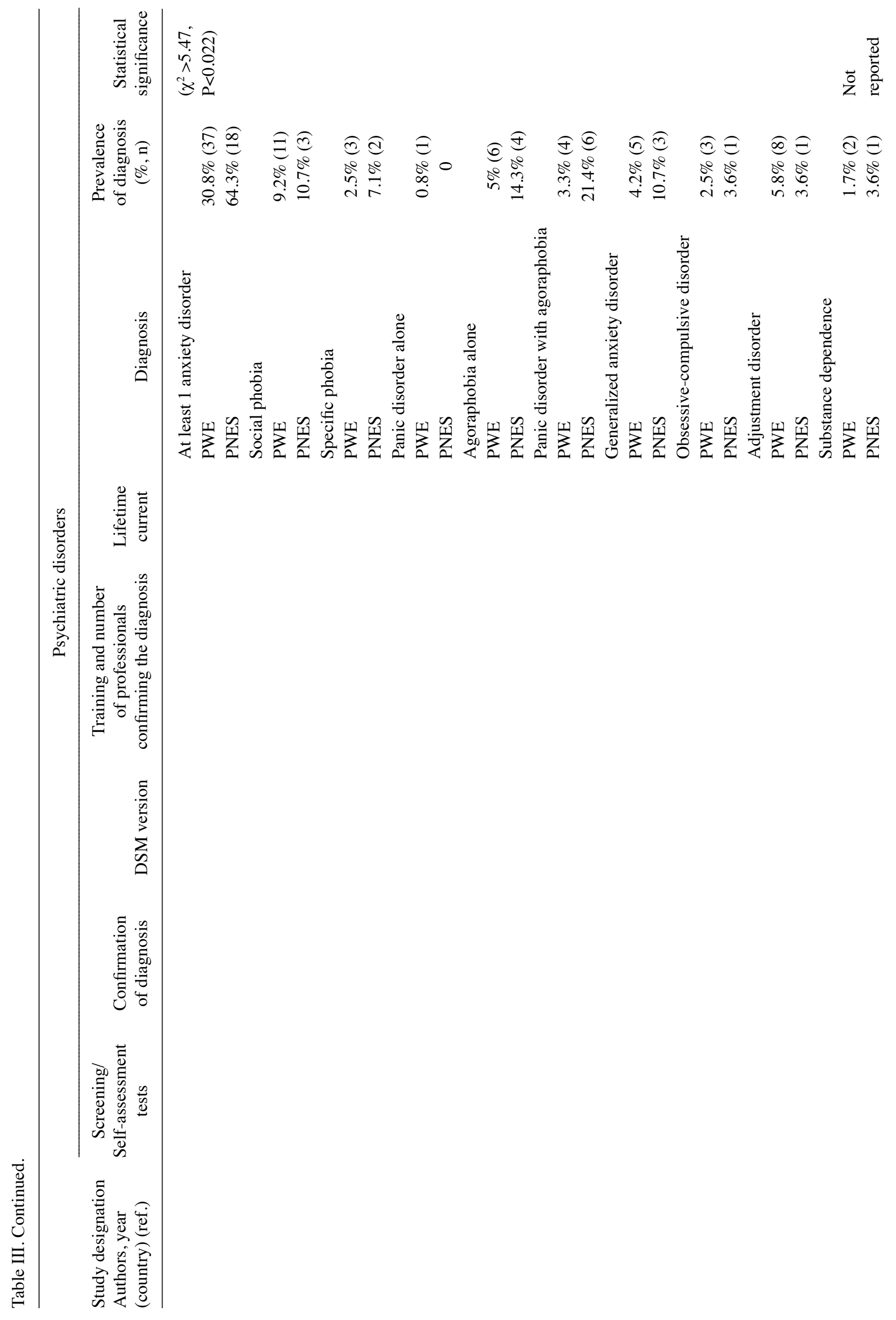




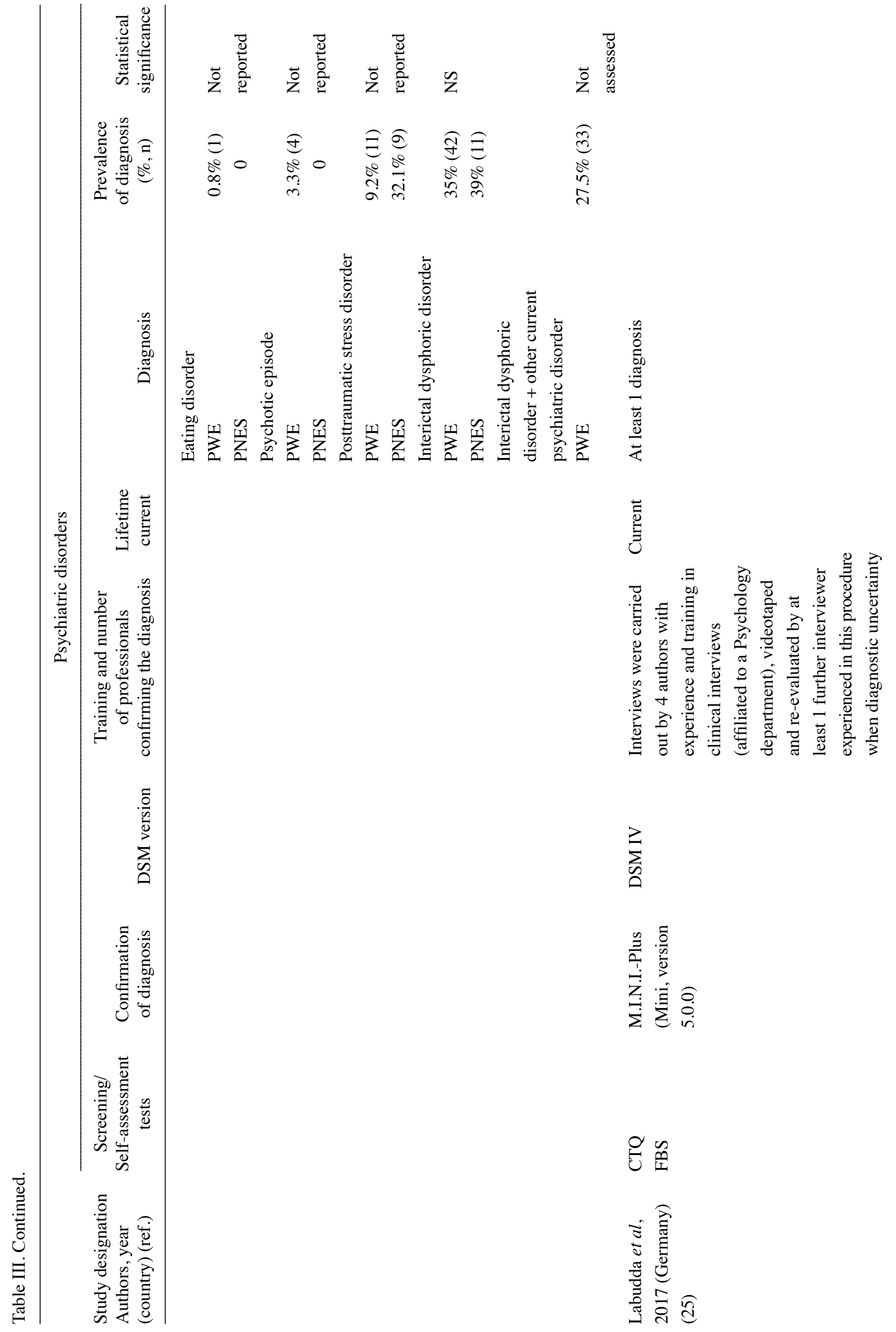




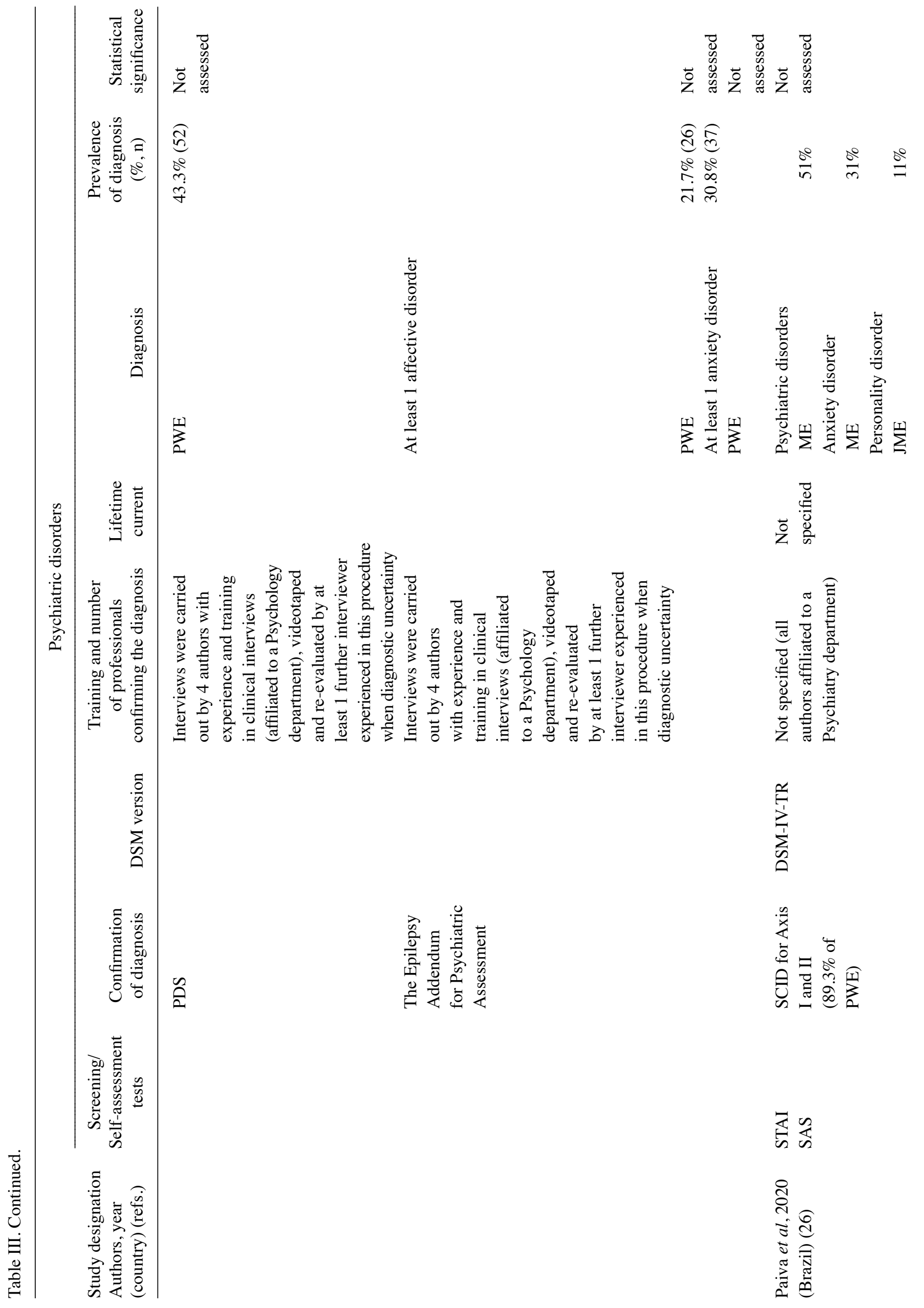




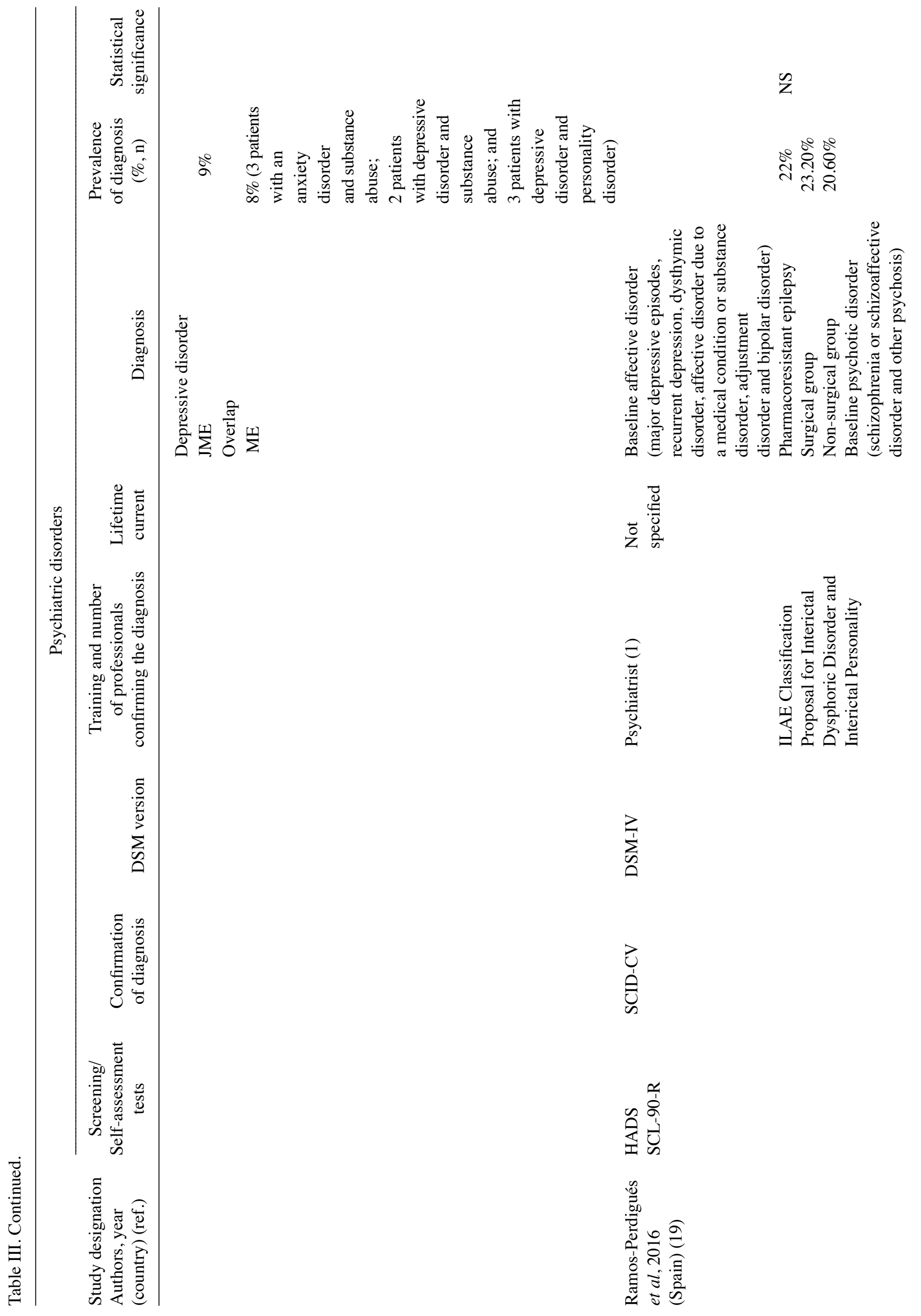


GURGU et al: PSYCHIATRIC COMORBIDITIES IN ADULT PATIENTS WITH EPILEPSY

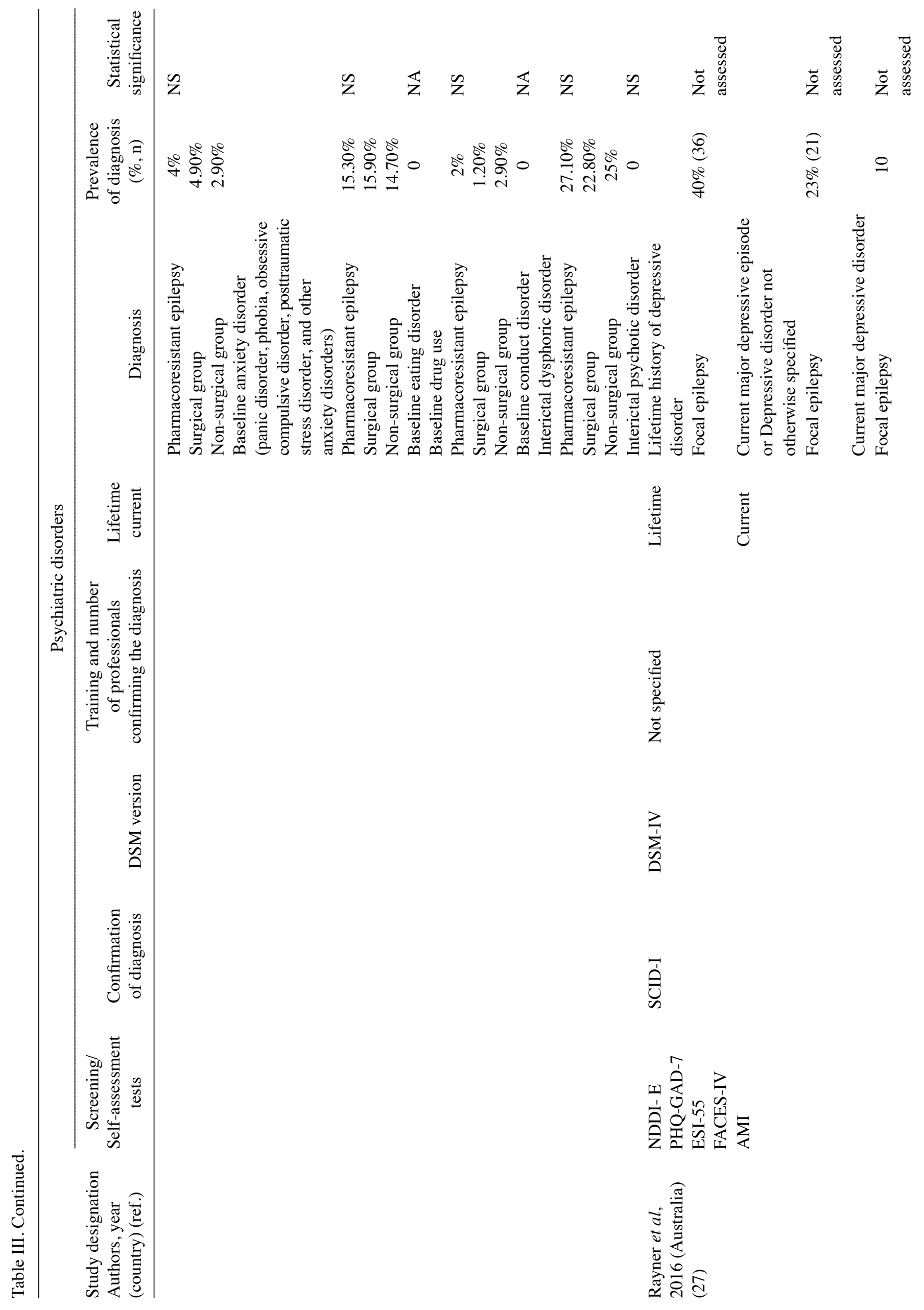




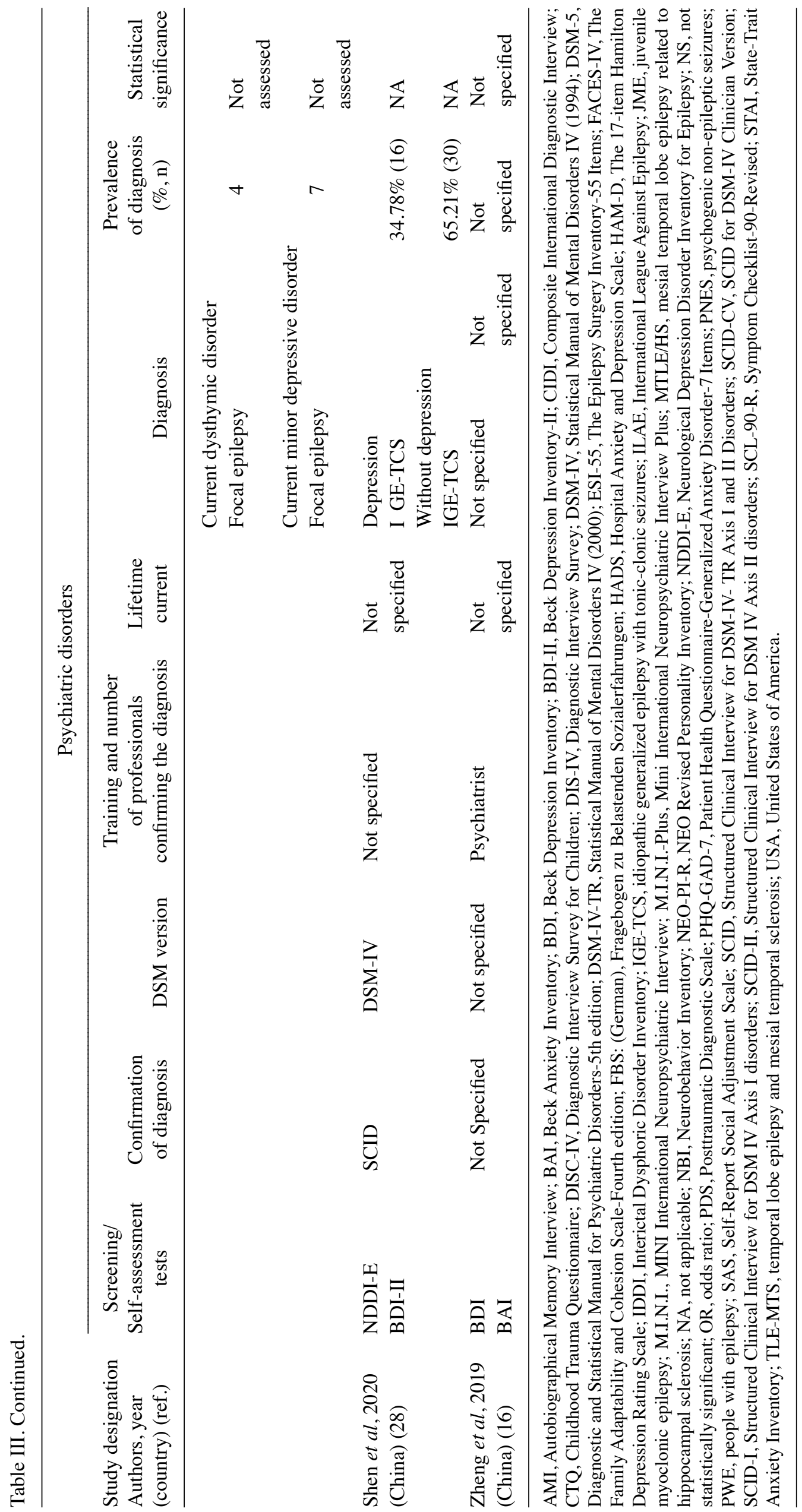


respectively, and statistical analysis (including comparison with siblings and external controls) showed no relation between suicidal thoughts and behaviors and epilepsy (17).

In a study that examined the relationship between white matter integrity [assessed by Diffusion Tensor Imaging (DTI)] and depression in a small group of patients with TLE and depression (major depressive disorder or dysthymic disorder, as per DSM-IV criteria) (23), the severity of the depression [The 17-item Hamilton Depression Rating Scale (HAM-D) score] was significantly and negatively correlated with the matter integrity in non-fronto-temporo-limbic areas [non-fronto-temporo-limbic fractional anisotropy (non-FTL-FA) composite score, $\mathrm{P}<0.05]$ and showed a similar trend for fronto-temporo-limbic areas $(\mathrm{P}=0.06)$, while in the control group (patients with depression, without epilepsy) the correlation was not statistically significant.

When the contribution of depression (major depressive disorder, dysthymic disorder) to executive functioning was assessed in TLE patients, it was observed that epilepsy patients with depression (not specified if current or lifetime) performed worse than epilepsy patients without depression in verbal fluency test for category switching $(\mathrm{P}=0.04)(22)$.

One study investigated two possible symptom-based phenotypes of depression (named by authors cognitive and somatic, respectively) in focal epilepsy (27). For the cognitive depression group, the authors described higher rates of parasuicidal or suicidal thoughts, feelings of worthlessness, delusions of guilt, dysphoric mood than in the somatic depression (but the intergroup comparison showed no statistical significance for these DSM-IV symptoms). When these patients were compared with non-depressed epilepsy patients; they were more likely to have a left-lateralized seizure focus $(\mathrm{P}=0.034)$, but there were no other differences in any other epileptological variables or in anticonvulsant or psychotropic pharmacotherapy. Compared with healthy subjects, patients with cognitive depression exhibited significantly reduced semantic and episodic autobiographic memory across all life periods (including significantly worse overall semantic and episodic recollection) and significantly reduced delayed recall across auditory-verbal $(\mathrm{P}=0.032)$ and visual domains $(\mathrm{P}=0.002)$ on the Wechsler Memory Scale-IV (WMS-IV) subtests, in the context of intact immediate learning $(\mathrm{P}>0.050)$. Instead, somatic depression patients presented significant higher rates of somatic symptoms (appetite changes, sleep changes) and anhedonia (for all $\mathrm{P}<0.01$ ) and there were no differences when compared with epilepsy patients without depression in any other epileptological variables or in anticonvulsant or psychotropic pharmacotherapy. Somatic depression patients had more seizures and greater variability in seizure frequency than the cognitive phenotype (monthly average seizure frequency 17.5 and 4, respectively) and performed worse than healthy subjects in childhood episodic, early adulthood semantic, overall episodic, and delayed visual recall, but these inter-group differences were not statistically significant. Odds ratio (OR) analysis suggested that, compared with patients with somatic depression, depressed patients with the cognitive phenotype were more likely to have semantic autobiographic memory deficit (i.e., Autobiographical Memory Interview (AMI) subscale score b 50; 95\% CI=0.162-81.705), episodic autobiographic memory deficit (i.e., AMI subscale score b 16; 95\% CI=0.316-16.512) and impaired immediate verbal learning (i.e., WMS-IV score $\leq 8 ; 95 \%$ ). Psychosocial and demographic features of the two phenotypes of depression [age, relationship status, level of depressive symptoms (NDDI-E), level of family satisfaction (The Family Adaptability and Cohesion Scale-Fourth edition (FACES-IV), symptoms of anxiety (PHQ-GAD-7), quality of life (The Epilepsy Surgery Inventory-55 Items (ESI-55) or psychotropic medications] were not significantly different between groups ( $\mathrm{P}>0.05$ for all comparisons) (27).

In a study assessing brainstem echogenicity in depressed patients with IGE-TCS there was no significant difference in third ventricle width between epilepsy patients with and without depression $(\mathrm{P}=0.293)$, but more epilepsy patients with depression exhibited hypoechogenic brainstem raphe $(\mathrm{P}=0.000)(28)$.

Interictal dysphoric disorder is a psychiatric disorder assumed to be specific for epilepsy patients. Prevalence of interictal dysphoric disorder was $35 \%$ in a general population of epilepsy patients (associated in $27 \%$ of the total cases with mood disorder, anxiety disorder, both mood and anxiety disorder, substance abuse), $39 \%$ in a population with psychogenic non-epileptic seizure (both epilepsy patients and psychogenic non-epileptic seizure patients were assessed in a tertiary centre and comparison between the two groups did not show a statistical difference) (24), 18.4\% in resistant TLE-MTS (significantly higher than in healthy subjects from the control group) (21) and $27.1 \%$ in a group of patients with pharmacoresistant epilepsy (also significantly higher than in the control group) (19). In a study of prevalence of interictal dysphoric disorder in epilepsy patients it was demonstrated that epilepsy patients with interictal dysphoric disorder had more frequently than epilepsy patients without interictal dysphoric disorder at least one current psychiatric disorder $\left(\chi^{2}=32.68, \mathrm{P}<0.001\right)$, at least one current affective disorder [major depressive episode, recurrent depressive disorder, dysthymia $\left.\left(\chi^{2}=25.64, \mathrm{P}<0.001\right)\right]$ and at least one current anxiety disorder [social phobia, specific phobia, panic disorder alone, agoraphobia alone, panic disorder with agoraphobia, generalized anxiety disorder (GAD), obsessive compulsive disorder, adjustment disorder $\left.\left(\chi^{2}=17.35, \mathrm{P}<0.001\right)\right]$, and that $66 \%$ of epilepsy patients without a current psychiatric comorbidity had a diagnosis of a past psychiatric disorder (recurrent depressive disorder currently remitted and substance addiction) (24). Also, epilepsy patients with interictal dysphoric disorder and other psychiatric disorders had similar symptoms with epilepsy patients with interictal dysphoric disorder alone (euphoria, Fisher's exact test: $\mathrm{P}=1.0$; irritability, Fisher's exact test: $\mathrm{P}=0.698$ ) (24). In patients with drug-resistant epilepsy assessed for surgery, the presence of interictal dysphoric disorder was correlated with epilepsy location: $33.3 \%$ of patients had no loci established, $8.3 \%$ had extratemporal loci, and $58.3 \%$ had temporal loci $(\mathrm{P}=0.002)(19)$. Analysis of the diagnostic superposition between interictal dysphoric disorder and DSM Axis I psychiatric disorders in patients with TLE-MTS showed that none of the interictal dysphoric disorder patients and none of the healthy subjects in the control group had a DSM Axis I psychiatric disorder (21). Multivariate logistic regression analysis revealed that the significant risk factors for interictal dysphoric disorder in patients with pharmacoresistant TLE-MTS were the presence of left-sided mesial temporal sclerosis $(\mathrm{OR}=3.22$; 
$\mathrm{P}=0.008)$, previous psychiatric treatment $(\mathrm{OR}=4.29 ; \mathrm{P}=0.007)$ and the use of more than one antiepileptic drug $(\mathrm{OR}=2.73$; $\mathrm{P}=0.02)(21)$.

Anxiety disorders. Current anxiety disorders (anxiety disorder, panic disorder with or without agoraphobia, agoraphobia without panic disorder, social phobia during the previous year) had a prevalence of $6.3 \%$ among adult patients with childhood onset epilepsy. In this case the prevalence of current anxiety disorders was not statistically significant different between cases and siblings, but when cases were compared with external controls, current anxiety disorders were associated with a significantly decreased odds of epilepsy (17). At least one anxiety disorder was present in $30.8 \%$ of a sample of epilepsy patients from a tertiary referral centre (social phobia in $9.2 \%$, specific phobia in $2.5 \%$, panic disorder alone in $0.8 \%$, agoraphobia alone in $5 \%$, panic disorder with agoraphobia in $3.3 \%$, GAD in $4.2 \%$ and adjustment disorder in $5.8 \%$ ). The overall comparison with a group of patients with psychogenic non-epileptic seizures showed that for all disorders mentioned above (except adjustment disorder), the prevalence was higher in the psychogenic non-epileptic seizures group (24).

Lifetime anxiety disorders (generalized anxiety disorder, panic disorder with and without agoraphobia, agoraphobia, agoraphobia without panic disorder, social phobia, and specific phobia) were present in $15.6 \%$ of adults with childhood onset epilepsy. As in the case of current anxiety disorders, in this study the prevalence of lifetime anxiety disorders was not significantly different between cases and siblings, but was significantly higher in the external controls than in epilepsy patients (17).

Where lifetime or current was not specified, anxiety disorder was present in 19 and $31 \%$, respectively in two populations of patients with JME $(20,26)$, in $7 \%$ of the post-surgical patients with MTLE/HS (20) and in $15.3 \%$ of patients with pharmacoresistant epilepsy (diagnosed with panic disorder, phobia, obsessive compulsive disorder, posttraumatic stress disorder, and other anxiety disorders) (19). Prevalence of generalized anxiety disorder in pharmacoresistant TLE-MTS was $15.78 \%$ (significantly higher than in healthy controls, $\mathrm{P}=0.001$ ) (21).

In patients with JME, the presence of anxiety disorder (not specified if current or lifetime) was moderately correlated with a higher frequency of generalized tonico-clonic seizures (GTCS) $(\tau=-0.320 ; \mathrm{P}=0.008)$ and with worse familiar relationship adjustment [Social Adjustment Scale (SAS) family score, $(\tau=0.241 ; \mathrm{P}=0.025)]$. There was no other significant correlation between the presence of anxiety disorder and other social adjustment domains (SAS scores for work, leisure/social, economic or total) (26).

Psychotic disorders. Only four studies reported the prevalence of psychotic disorders in epilepsy patients. The prevalence of lifetime psychosis (ictal or interictal), as reported historically by patients during their first psychiatric assessment in epilepsy centre was $2.5 \%$ in a general epilepsy patient population $(2,568$ epilepsy patients screened), $4.3 \%$ in focal idiopathic epilepsy and even higher in TLE (8.5\%). In this specific study, interictal psychosis was retrospectively diagnosed in $4.12 \%$ of the focal idiopathic epilepsy cases (18).

When DSM criteria were used, $3.3 \%$ of the epilepsy patients from a tertiary epilepsy centre were diagnosed with current psychotic episode (24). Psychotic disorders (schizophrenia, schizoaffective disorder, other psychosis) had a prevalence of $4 \%$ in pharmacoresistant epilepsy (19) and interictal psychosis had a prevalence of 5\% in pharmacoresistant TLE-MTS (not significant in comparison with controls) (21). Interictal psychotic disorder (as per ILAE proposed criteria) was not diagnosed in any of the patients with drug resistant epilepsy assessed for surgery eligibility (19).

The only study that specifically investigated the presence of psychosis in focal epilepsy without central nervous system diseases found a significant difference between the prevalence of psychosis (predominantly interictal, but also peri-ictal) between patients with late-onset epilepsy (at $>49$ years) and patients with early onset epilepsy (at $<49$ years), basically with no cases in the old-onset group $(\mathrm{P}=0.016)$. Significant determinants associated with psychosis in this study were duration of illness $(\mathrm{P}=0.000001)$ and presence of temporal lobe epilepsy $(\mathrm{P}=0.000343)$, while sex and age at examination were not contributory to psychosis (18).

Personality disorders. Personality disorders (assessed using DSM criteria) were present in $8 \%$ and respectively $11 \%$ of the JME patients assessed in two studies $(20,26)$ and in none of the post-surgical MTLE/HS cases assessed in one study (20).

A study that specifically investigated personality profile in JME and post-surgical MTLE/HS patients using NBI and NEO-PI-R found a positive correlation between the neuroticism domain of NEO-PI-R and psychiatric symptoms in both MTLE/HS ( $\mathrm{r}=0.201 ; \mathrm{P}=0.003)$ and JME $(\mathrm{r}=0.145 ; \mathrm{P}=0.010)$ groups. Also, post-surgical MTLE-HS patients with psychiatric diagnoses (depressive disorders, anxiety disorders and personality disorders), had higher total NBI scores than those with no psychiatric symptoms $(\mathrm{P}<0.001)$ and JME patients with psychiatric diagnoses and uncontrolled GTCS had higher total NBI scores than those with no psychiatric symptoms/controlled GTCS $(\mathrm{P}=0.001$ and $\mathrm{P}=0.032$, respectively). In patients with psychiatric symptoms, $13 / 20 \mathrm{NBI}$ traits $(65 \%)$ were higher in the MTLE/HS group than in the JME group $(9 / 20,45 \%)(\mathrm{P}=0.05)(20)$.

Interictal personality, a behavioral condition that could be associated with epilepsy (assessed with NBI) had a prevalence of $37.9 \%$ in pharmacoresistant TLE (significantly higher than in controls) (21). When analysis of the diagnostic superposition between interictal personality and DSM Axis I psychiatric disorders was conducted, 15 (41.6\%) of the epilepsy patients with interictal personality had a DSM Axis I psychiatric disorder (major depressive disorder 66.6\%, interictal psychosis $18.7 \%$, conversion disorder $13.4 \%$ ), while none of the subjects in the control group with DSM Axis I psychiatric disorders demonstrated superposition with interictal personality. Multivariate logistic regression models showed that significant risk factors for interictal personality are the presence of bilateral mesial temporal sclerosis $(\mathrm{OR}=3.27 ; \mathrm{P}=0.008)$, longer disease duration $(\mathrm{OR}=3.39 ; \mathrm{P}=0.006)$ and the presence of major depressive disorder (21).

Other important associations. In a study assessing the prevalence of interictal dysphoric disorder and interictal personality, significantly more patients with drug-resistant TLE-MTS had a history of previous psychiatric treatment $(\mathrm{P}=0.001)$, family 
history of epilepsy ( $\mathrm{P}=0.001)$, and family history of psychiatric disorders $(\mathrm{P}=0.001)(21)$.

Current psychiatric disorders in a general population of epilepsy patients are associated with childhood maltreatment experiences (25). Patients with current psychiatric disorders [major depressive episode, recurrent depressive disorder, dysthymia, social phobia, specific phobia, panic disorder alone, agoraphobia alone, panic disorder with agoraphobia, generalized anxiety disorder, obsessive-compulsive disorder, adjustment disorder, substance dependence, eating disorder, psychotic episode-diagnosed as per DSM IV criteria or posttraumatic stress disorders-diagnosed with Posttraumatic Diagnostic Scale (PDS)] had suffered significantly more childhood maltreatment experiences [higher Childhood Trauma Questionnaire (CTQ) scores-total score ( $\mathrm{t}=3.41, \mathrm{P}=0.001)$, emotional abuse $(\mathrm{t}=2.83, \mathrm{P}=0.006)$ and emotional neglect $(\mathrm{t}=3.47, \mathrm{P}=0.001)]$; had higher Fragebogen $\mathrm{zu}$ Belastenden Sozialerfahrungen (FBS) scores $(\mathrm{t}=3.75, \mathrm{P}<0.001)$ and significantly more reported severe emotional abuse $\left(\chi^{2}=13.72\right.$, $\mathrm{P} \leq 0.001)$, emotional neglect $\left(\chi^{2}=12.28, \mathrm{P} \leq 0.001\right)$, and physical abuse $\left(\chi^{2}=3.98, P=0.046\right)$. Age at epilepsy onset, epilepsy duration, number of different seizure types and number of patients in each seizure frequency category did not differ between epilepsy patients with psychiatric disorders and epilepsy patients without psychiatric disorders. Direct logistic regression showed that CTQ total score, FBS score and epilepsy-related variables, i.e., age of epilepsy onset, epilepsy duration, number of seizure types and seizure frequency, were all predictors for a current psychiatric disorder $\left[\chi^{2}(9,120)=27.13, \mathrm{P}<0.001\right.$, Nagelkerke $\left.R^{2}=0.28\right)(25)$.

In a prospective case-control study, designed to evaluate the psychiatric outcome in drug-resistant epilepsy patients that underwent surgery, compared with those who did not, existing differences in baseline affective disorders (major depressive episodes, recurrent depression, dysthymic disorder, affective disorder due to a medical condition or substance disorder, adjustment disorder and bipolar disorder), psychotic disorders (schizophrenia or schizoaffective disorder and other psychosis), anxiety disorders (panic disorder, phobia, obsessive compulsive disorder, posttraumatic stress disorder, and other anxiety disorders), eating disorder, drug use, conduct disorder (diagnosed as per DSM-IV, not specified if current or lifetime), interictal dysphoric disorder and interictal psychotic disorder (diagnosed as per ILAE Classification Proposal) did not depended on the presence vs. absence of complex partial seizures (CPS), the cerebral hemisphere involved (left vs. right), and seizure localization (temporal vs. extratemporal vs. unestablished). Paired comparisons between baseline and 6 months after surgery showed no statistical change in all psychiatric disorders mentioned above in any group (19).

\section{Discussion and limitations}

This systematic review examined whether current literature provides quality information on the prevalence and risk factors for psychiatric disorders in epilepsy patients. In order to properly treat epilepsy patients with psychiatric comorbidities, it is very important to have an accurate psychiatric diagnosis, in patients who are usually attended by neurologists or epileptologists. Although in recent years numerous screening tools have been developed and validated for the screening of anxiety disorders (29) and depressive disorders (30-33), in epilepsy patients, these instruments cannot replace a formal psychiatric assessment (34). Unfortunately, most recent studies reporting data on psychiatric comorbidities in epilepsy used these type of instruments and, as a result, only psychiatric symptoms associated with epilepsy were reported and analyzed.

The overall reported prevalence of mood/affective disorders was 12.8 to $34.78 \%$, while the prevalence of specific diagnoses as per DSM-IV is difficult to assess. In general, a higher prevalence of mood/affective disorders was reported when the temporal characteristics (lifetime vs. current) were not specified. Current and lifetime mood disorders appear to be less frequently encountered in IGE and more prevalent in focal drug-resistant epilepsy (mainly TLE). In TLE, the severity of depression was found to be associated with white matter anomalies in non-fronto-temporo-limbic areas and with cognitive impairment (executive function). In focal epilepsies, cognitive depression was found to be associated with a left-lateralized seizure focus (no other associations were found between depression and epileptological variables, antiepileptic drugs or psychotropic treatments in epilepsy patients with or without depression) and with cognitive impairment (semantic and autobiographic memory, delayed auditory-verbal and visual recall). In IGE, depression was associated with hypoechogenic brainstem raphe (without any difference on third ventricle width between epilepsy patients with or without depression).

Suicidal risk was reported only for adults with childhood onset epilepsy and in this population, it beared no significant relation with epilepsy. This risk was lower than previously reported (35), but the childhood onset epilepsies often have a more favorable course, with remission in adulthood.

Interictal dysphoric disorder is a highly controversial epilepsy-specific psychiatric disorder, that is not recognized by DSM but has specific diagnosis criteria developed by epileptologists (36). Its prevalence ranged between $18.4 \%$ in resistant TLE-MTS to $35 \%$ in a general population of patients with epilepsy. The results of two studies are contrasting: In a general sample of epilepsy patients, interictal dysphoric disorder was more frequently present when other psychiatric disorders were present and interictal dysphoric disorder symptoms frequencies were similar in patients with or without interictal dysphoric disorder, while in resistant TLE-MTS none of the patients with interictal dysphoric disorders had an DSM Axis I psychiatric disorder $(21,24)$. In drug-resistant TLE, the risk factors for interictal dysphoric disorder were left-sided MTS, previous psychiatric treatment and the use of more than one antiepileptic drug. In drug-resistant epilepsies assessed for surgery, the prevalence of interictal dysphoric disorder was higher in TLE patients as well.

Prevalence of anxiety disorders was between $5.6 \%$ in adults with childhood onset epilepsy and $30.8 \%$ in a sample from a general population of epilepsy patients. As in the case of mood/affective disorders, the prevalence of specific diagnoses and temporal characteristics were hard to systemize, but current anxiety disorders appeared to have a higher prevalence. We speculate that this trend is related to the long duration of epilepsy and to the higher severity of seizures in selected studies. In JME, the presence of anxiety disorders was correlated with a higher frequency of GTCS and with worse 
familial adjustment (no other correlations were found between the presence of anxiety disorder and other social adjustments domains such as work, leisure/social or economic).

Psychotic disorders were found to have a low prevalence across all epilepsy types (2.5-5\%), but only four studies reported data on this psychiatric disorder $(18,19,21,24)$. Prevalence of psychosis (ictal, interictal) was significantly higher in patients with early-onset focal epilepsy and its presence was corelated with longer disease duration and presence of TLE (sex and age at examination were not contributory to psychosis).

Prevalence of personality disorders was assessed in three studies $(20,21,26)$. Personality disorders, under these circumstances, appear to be a characteristic of JME (but only one study assessed patients with post-surgical MTLE/HS). When JME, MTLE/HS and control patients were compared, no significant differences were found regarding normal personality traits or supposed interictal personality characteristics between groups.

Interictal personality (also known as Gastaut-Geschwind Syndrome) (37), another disorder described only by epileptologists, had a high prevalence in pharmacoresistant TLE (37.9\%), but was not confirmed when DSM-IV criteria were used. Instead significant correlations were found between presence of interictal personality and presence of bilateral MTS, longer disease duration and presence of major depressive disorder.

Another interesting correlation that was made in epilepsy patients is the association between childhood maltreatment experiences, age at epilepsy onset, epilepsy duration, seizure types, seizure frequency and the presence of any current DSM Axis I psychiatric disorder. Another study showed that the presence of DSM Axis I psychiatric disorders, interictal dysphoric disorder and interictal psychotic disorder in pharmaco-resistant epilepsy do not depend on the presence vs. absence of complex partial seizures, lateralization or localization of seizures.

The last study was the only study that assessed the presence of psychiatric disorders diagnosed per DSM-IV in pharmacoresistant epilepsies that did not undergo surgery at baseline and at six months and found that there were no significant diagnosis changes over a period of 6 months (19).

The prevalence of psychiatric disorders in our analysis resemble to some extent the prevalence reported in a non-systematic review of literature published in 2004 (38), but direct comparison is not possible because both epilepsy and psychiatric disorder classifications have changed since its publication.

Our analysis is subject to many limitations. Some important gaps are present in recent literature. We were not able to find eligible studies on psychiatric disorders in patients with single unprovoked seizures or in patients with a recent diagnosis of epilepsy. Methodology used in the studies selected for this analysis was highly heterogenous and this did not allow us to perform a meta-analysis. Most studies included small samples of epilepsy patients and were conducted in epilepsy units, thus introducing biases such as exclusion of patients with severe neurological pathologies, over-representation of TLE, over-representation of pharmacoresistant epilepsy and exclusion of patients with less severe epilepsy. Control groups were smaller than patient samples in eight studies and six studies used control groups with other pathologies. Formal psychiatric assessment was not performed in controls in 7 studies. In almost $40 \%$ of the studies, the epilepsy diagnosis was not formulated very clearly. Epilepsy duration was not specified in three articles, seizure frequency was not specified in three articles and antiepileptic drug treatment was not specified in four articles. Even if most studies included epileptic subtypes in their general sample, the authors did not stratify the results by subtype. Only four studies report that psychiatric assessment was performed by qualified professionals. Three versions of the DSM and nine versions of structured or semi-structured clinical interviews were used to diagnose the psychiatric disorders. Finally, in eight studies we are not sure if the psychiatric disorders reported are current or lifetime disorders. Specific psychiatric disorder diagnoses are not mentioned in five studies (only DSM broader category is provided). As is the case for epilepsy subtypes, even when specific psychiatric disorders are reported, the authors did not stratify results by subtype in many cases.

\section{Conclusions}

The studies included in our analysis are heterogenous. Objectives, methodology and result reporting were found to be different in all studies. Psychiatric disorders are present in a high proportion of epilepsy patients, at least at some time point in the disease evolution.

Prevalence of any psychiatric disorder observed was up to $51 \%$ in IGE, up to $43.1 \%$ in TLE, up to $43.3 \%$ in a general population of patients with epilepsy. The most frequent psychiatric comorbidities of epilepsy are mood/affective disorders (up to $40 \%$ for lifetime occurrence and up to $23 \%$ for current occurrence), anxiety disorders (up to $30.8 \%$ for lifetime occurrence and up to $15.6 \%$ for current occurrence), personality disorders (up to $11 \%$ in JME) and psychotic disorders (up to $4 \%$ of patients with epilepsy). Personality disorders and anxiety disorders are more frequently described (and studied) in IGE patients, while depressive disorders and psychotic disorders are more frequently described in focal epilepsy (mainly TLE) and pharmacoresistant epilepsy. Evidence regarding the presence and prevalence of interictal dysphoric disorder and interictal personality is contradictory.

Depressive disorders might be associated with specific brain imaging findings and with cognitive impairment in focal epilepsy. Anxiety disorders are associated with higher frequency of generalized tonico-clonic seizures and with worse social functioning. Psychotic disorders are associated with longer duration of epilepsy. Childhood maltreatment experiences are a powerful predictor for the occurrence of psychiatric comorbidities in epilepsy patients, while data regarding association of other epilepsy characteristics (age at epilepsy onset, epilepsy duration, seizure type, seizure localization and lateralization, seizure frequency) with the presence of psychiatric disorders are conflicting.

Future research in this area should assess the presence of psychiatric disorders in newly diagnosed epilepsy/after a single unprovoked seizure, should include a detailed sociodemographic description for all patients/controls, should use standardized assessment tools (including standardized epilepsy and seizure classification, standardized reporting for 
seizure frequency-as recommended by ILAE for clinical practice, standardized structured clinical interview for diagnosis of psychiatric disorders) and should stratify data according to the specific epilepsy and psychiatric diagnosis.

\section{Acknowledgements}

Not applicable.

\section{Funding}

No funding was received.

\section{Availability of data and materials}

All information provided in this review is documented by relevant references.

\section{Authors' contributions}

RSG conceived the research protocol (including search strategy and study inclusion/exclusion criteria), cross-checked all full articles reviewed and selected by RID, cross-checked data extracted from the selected studies, performed the quality assessment of studies included, prepared the manuscript and modified the manuscript as per the feedback received from AMC and CAP. AMC reviewed the methodology and the manuscript, provided feedback, submitted the manuscript and the cover letter. RID searched the databases, screened title and abstracts, identified and excluded duplicate titles, assessed the full text articles for eligibility, excluded articles that did not meet inclusion criteria or meet the exclusion criteria and extracted relevant data from selected studies. CAP reviewed the methodology and the manuscript and provided feedback. All authors read and approved the final manuscript for publication.

\section{Ethics approval and consent to participate}

Not applicable.

\section{Patient consent for publication}

Not applicable.

\section{Competing interests}

The authors declare that they have no competing interests.

\section{Authors' information}

Adela Magdalena Ciobanu: ORCID: ID0000-0003-2520-5486.

\section{References}

1. World Health Organization (WHO): The ICD-10 classification of mental and behavioural disorders: Clinical descriptions and diagnostic guidelines. World Health Organization, 1992.

2. Salpekar JA and Mula M: Common psychiatric comorbidities in epilepsy: How big of a problem is it? Epilepsy Behav 98: 293-297, 2019.
3. Michaelis R, Tang V, Goldstein LH, Reuber M, LaFrance WC Jr, Lundgren T, Modi AC and Wagner JL: Psychological treatments for adults and children with epilepsy: Evidence-based recommendations by the international league against epilepsy psychology task force. Epilepsia 59: 1282-1302, 2018.

4. Vogt VL, Äikiä M, Del Barrio A, Boon P, Borbély C, Bran E, Braun K, Carette E, Clark M, Cross JH, et al: Current standards of neuropsychological assessment in epilepsy surgery centers across Europe. Epilepsia 58: 343-355, 2017.

5. Wilson SJ, Baxendale S, Barr W, Hamed S, Langfitt J, Samson S, Watanabe M, Baker GA, Helmstaedter C, Hermann BP and Smith ML: Indications and expectations for neuropsychological assessment in routine epilepsy care: Report of the ILAE neuropsychology task force, diagnostic methods commission, 2013-2017. Epilepsia 56: 674-681, 2015.

6. Scheffer IE, Berkovic S, Capovilla G, Connolly MB, French J, Guilhoto L, Hirsch E, Jain S, Mathern GW, Moshé SL, et al: ILAE classification of the epilepsies: Position paper of the ILAE commission for classification and terminology. Epilepsia 58: 512-521, 2017.

7. Fabiano F and Haslam N: Diagnostic inflation in the DSM: A meta-analysis of changes in the stringency of psychiatric diagnosis from DSM-III to DSM-5. Clin Psychol Rev 80: 101889, 2020.

8. Lacey CJ, Salzberg MR and D'Souza WJ: Risk factors for depression in community-treated epilepsy: Systematic review. Epilepsy Behav 43: 1-7, 2015.

9. Gandy M, Sharpe L and Perry KN: Psychosocial predictors of depression and anxiety in patients with epilepsy: A systematic review. J Affect Disord 140: 222-232, 2012.

10. Mula M: Developments in depression in epilepsy: Screening, diagnosis, and treatment. Expert Rev Neurother 19: 269-276, 2019.

11. Wang Z, Luo Z, Li S, Luo Z and Wang Z: Anxiety screening tools in people with epilepsy: A systematic review of validated tools. Epilepsy Behav 99: 106392, 2019.

12. Moher D, Liberati A, Tetzlaff J and Altman DG; PRISMA Group: Preferred reporting items for systematic reviews and meta-analyses: The PRISMA statement. PLoS Med 6: e1000097, 2009.

13. Sterne JAC, Savović J, Page MJ, Elbers RG, Blencowe NS, Boutron I, Cates CJ, Cheng HY, Corbett MS, Eldridge SM, et al: RoB 2: A revised tool for assessing risk of bias in randomised trials. BMJ 366: 14898, 2019.

14. Wells GA, Shea B, O'Connell D, Peterson J, Welch V, Losos M and Tugwell P: The Newcastle-Ottawa Scale (NOS) for assessing the quality if nonrandomized studies in meta-analyses. 2015. http://www.ohri.ca/programs/clinical_epidemiology/oxford.asp. Accessed March 14, 2021.

15. The Joanna briggs institute critical appraisal tools for use in JBI systematic reviews: Checklist for analytical cross-sectional studies. 2017. https://jbi.global/critical-appraisal-tools. Accessed March 14, 2021.

16. Zheng Y, Ding X, Guo Y, Chen Q, Wang W, Zheng Y, Wang S, Ding $Y$ and Ding M: Multidisciplinary management improves anxiety, depression, medication adherence, and quality of life among patients with epilepsy in eastern China: A prospective study. Epilepsy Behav 100: 106400, 2019.

17. Baldin E, Hesdorffer DC, Caplan R and Berg AT: Psychiatric disorders and suicidal behavior in neurotypical young adults with childhood-onset epilepsy. Epilepsia 56: 1623-1628, 2015.

18. Konishi R and Kanemoto K: Psychosis rarely occurs in patients with late-onset focal epilepsy. Epilepsy Behav 111: 107295, 2020.

19. Ramos-Perdigués S, Baillés E, Mané A, Carreño M, Donaire A, Rumia J, Bargalló N, Boget T, Setoain X, Valdes M and Pintor L: A prospective study contrasting the psychiatric outcome in drug-resistant epilepsy between patients who underwent surgery and a control group. Epilepsia 57: 1680-1690, 2016.

20. Alonso NB, de Albuquerque M, Vidal-Dourado M, Cavicchioli LH, Mazetto L, de Araújo Filho GM, de Figueiredo Ferreira Guilhoto LM, Centeno RS and Yacubian EMT: Revisiting personality in epilepsy: Differentiation of personality in two epilepsies starting in adolescence. Epilepsy Behav 97: 75-82, 2019.

21. de Araújo Filho GM, Tarifa B, Santos RE, de Oliveira Dias AL, Ulliano JRL and Marques LHN: Clinical and sociodemographic variables associated with interictal dysphoric disorder and interictal personality in patients with drug-resistant temporal lobe epilepsy: A controlled study. Epilepsy Behav 69: 100-103, 2017.

22. Galioto R, Tremont G, Blum AS, LaFrance WC Jr, Crook CL and Davis JD: Depressive symptoms contribute to executive deficits in temporal lobe epilepsy. J Neuropsychiatry Clin Neurosci 29: 135-141, 2017. 
23. Kavanaugh B, Correia S, Jones J, Blum A, LaFrance WC Jr and Davis JD: White matter integrity correlates with depressive symptomatology in temporal lobe epilepsy. Epilepsy Behav 77: 99-105, 2017.

24. Labudda K, Illies D, Bien CG and Neuner F: Interictal dysphoric disorder: Further doubts about its epilepsy-specificity and its independency from common psychiatric disorders. Epilepsy Res 141: 13-18, 2018.

25. Labudda K, Illies D, Herzig C, Schröder K, Bien CG and Neuner F: Current psychiatric disorders in patients with epilepsy are predicted by maltreatment experiences during childhood. Epilepsy Res 135: 43-49, 2017.

26. Paiva ML, Lima EM, Siqueira IB, Rzezak P, Koike C, Moschetta SP, Vincentiis S, Alessi R, Khafif TC, Mendoza M and Valente KD: Seizure control and anxiety: Which factor plays a major role in social adjustment in patients with Juvenile myoclonic epilepsy? Seizure 80: 234-239, 2020.

27. RaynerG,JacksonGD andWilsonSJ:Two distinct symptom-based phenotypes of depression in epilepsy yield specific clinical and etiological insights. Epilepsy Behav 64: 336-344, 2016.

28. Shen J, Li DL, Tan XX, Tao WW, Xie CJ, Shi XG and Wang Y: A transcranial sonography study of brainstem and its association with depression in idiopathic generalized epilepsy with tonic-clonic seizures. Epilepsy Behav 102: 106589, 2020.

29. Micoulaud-Franchi JA, Lagarde S, Barkate G, Dufournet B, Besancon C, Trébuchon-Da Fonseca A, Gavaret M, Bartolomei F, Bonini $\mathrm{F}$ and McGonigal A: Rapid detection of generalized anxiety disorder and major depression in epilepsy: Validation of the GAD-7 as a complementary tool to the NDDI-E in a French sample. Epilepsy Behav 57: 211-216, 2016.

30. Lin CY and Pakpour AH: Using hospital anxiety and depression scale (HADS) on patients with epilepsy: Confirmatory factor analysis and rasch models. Seizure 45: 42-46, 2017.
31. Gur-Ozmen S, Leibetseder A, Cock HR, Agrawal N and von Oertzen TJ: Screening of anxiety and quality of life in people with epilepsy. Seizure 45: 107-113, 2017.

32. Rampling J, Mitchell AJ, Von Oertzen T, Docker J, Jackson J, Cock $\mathrm{H}$ and Agrawal N: Screening for depression in epilepsy clinics. A comparison of conventional and visual-analog methods. Epilepsia 53: 1713-1721, 2012.

33. Ciobanu AM, Roşca T, Vlădescu CM, Tihoan C, Popa C, Boer MC and Cergan R: Frontal epidural empyema (Pott's puffy tumor) associated with Mycoplasma and depression. Rom J Morphol Embryol 55 (Suppl 3): S1203-S1207, 2014.

34. Hesdorffer DC, Baldin E, Caplan R and Berg AT: How do we measure psychiatric diagnoses? Implications of the choice of instruments in epilepsy. Epilepsy Behav 31: 351-355, 2014.

35. Mula M, Kanner AM, Schmitz B and Schachter S: Antiepileptic drugs and suicidality: An expert consensus statement from the task force on therapeutic strategies of the ILAE commission on neuropsychobiology. Epilepsia 54: 199-203, 2013.

36. Amiri $M$ and Hansen CP: The interictal dysphoric disorder in patients with epilepsy: A doubtful disorder lacking diagnostic tools. Seizure 24: 70-76, 2015.

37. Trimble $M$ and Freeman A: An investigation of religiosity and the gastaut-geschwind syndrome in patients with temporal lobe epilepsy. Epilepsy Behav 9: 407-414, 2006.

38. Gaitatzis A, Trimble MR and Sander JW: The psychiatric comorbidity of epilepsy. Acta Neurol Scand 110: 207-220, 2004

(i) $\odot$ This work is licensed under a Creative Commons Attribution-NonCommercial-NoDerivatives 4.0 International (CC BY-NC-ND 4.0) License. 